\title{
MAGNESIUM ISOTOPE RATIOS IN $\omega$ CENTAURI RED GIANTS
}

\author{
G. S. Da Costa, John E. Norris, and David Yong \\ Research School of Astronomy and Astrophysics, Australian National University, Canberra, ACT 0200, Australia \\ Received 2013 February 23; accepted 2013 March 28; published 2013 April 30
}

\begin{abstract}
We have used the high-resolution observations obtained at the Anglo-Australian Telescope with Ultra-High Resolution Facility $(R \sim 100,000)$ and at Gemini-S with b-HROS $(R \sim 150,000)$ to determine magnesium isotope ratios for seven $\omega$ Cen red giants that cover a range in iron abundance from $[\mathrm{Fe} / \mathrm{H}]=-1.78$ to -0.78 dex, and for two red giants in M4 (NGC 6121). The $\omega$ Cen stars sample both the "primordial" (i.e., O-rich, Na- and Al-poor) and the "extreme" (O-depleted, Na- and Al-rich) populations in the cluster. The primordial population stars in both $\omega$ Cen and M4 show $\left({ }^{25} \mathrm{Mg},{ }^{26} \mathrm{Mg}\right) /{ }^{24} \mathrm{Mg}$ isotopic ratios that are consistent with those found for the primordial population in other globular clusters with similar $[\mathrm{Fe} / \mathrm{H}]$ values. The isotopic ratios for the $\omega$ Cen extreme stars are also consistent with those for extreme population stars in other clusters. The results for the extreme population stars studied indicate that the ${ }^{26} \mathrm{Mg} /{ }^{24} \mathrm{Mg}$ ratio is highest at intermediate metallicities $([\mathrm{Fe} / \mathrm{H}]<-1.4 \mathrm{dex})$, and for the highest $[\mathrm{Al} / \mathrm{Fe}]$ values. Further, the relative abundance of ${ }^{26} \mathrm{Mg}$ in the extreme population stars is notably higher than that of ${ }^{25} \mathrm{Mg}$, in contrast to model predictions. The ${ }^{25} \mathrm{Mg} /{ }^{24} \mathrm{Mg}$ isotopic ratio in fact does not show any obvious dependence on either $[\mathrm{Fe} / \mathrm{H}]$ or $[\mathrm{Al} / \mathrm{Fe}]$ nor, intriguingly, any obvious difference between the primordial and extreme population stars.
\end{abstract}

Key words: globular clusters: general - globular clusters: individual ( $\omega$ Centauri (NGC 5139), M4 (NGC 6121)) stars: abundances - stars: Population II

Online-only material: color figures

\section{INTRODUCTION}

The stellar system $\omega$ Centauri has been known to have an unusual stellar population for almost four decades. For example, there is now an extensive body of work which shows that, unlike the situation for most globular clusters, the member stars of $\omega$ Cen possess a large range in heavy element abundance together with distinctive element-to-iron abundance ratios (e.g., Johnson \& Pilachowski 2010; Marino et al. 2012, and references therein). Based on photometric and spectroscopic studies of lower mainsequence stars there is also evidence for a substantial spread in helium abundance in the cluster (e.g., Norris 2004; Piotto et al. 2005). Further, spectroscopic studies of stars near the mainsequence turnoff suggest that $\omega$ Cen has an age spread of order 2 Gyr (e.g., Pancino et al. 2011, and references therein). These properties, which are not shared, in toto, by any other globular cluster, have led to the suggestion that $\omega$ Cen is the nuclear remnant of a disrupted dwarf galaxy that was accreted by the Milky Way (e.g., Freeman 1993). Bekki \& Freeman (2003) have shown that this is dynamically plausible despite the tightly bound and retrograde current orbit of the cluster (Dinescu et al. 1999).

In one respect, however, $\omega$ Cen is very similar to the other globular clusters of the Milky Way and that is the presence of the so-called O-Na anti-correlation among the cluster stars (Norris \& Da Costa 1995a). It is now well established (e.g., Carretta et al. 2010) that essentially all Milky Way globular clusters show this phenomenon. It is characterized by the presence of two or three populations within a given globular cluster: primordial, intermediate and, in at least some clusters, an extreme population (e.g., Carretta et al. 2009). The primordial population shows abundances of the elements $\mathrm{C}, \mathrm{N}, \mathrm{O}, \mathrm{F}, \mathrm{Na}, \mathrm{Al}$, and $\mathrm{Mg}$ relative to $\mathrm{Fe}$ that are essentially equivalent to those for field halo stars with the same $[\mathrm{Fe} / \mathrm{H}]$ value. The intermediate population shows depletions in $\mathrm{O}$ of order $0.3 \mathrm{dex}$, and depletions in $\mathrm{C}$ and $\mathrm{F}$, which are coupled with enhancements in $\mathrm{N}, \mathrm{Na}$, and $\mathrm{Al}$. The extreme population shows even larger depletions in $\mathrm{O}$ and also depletions in the $\mathrm{Mg}$ abundance in addition to the $\mathrm{N}, \mathrm{Na}$, and $\mathrm{Al}$ enhancements.

The usual interpretation of these abundance correlations is that they are the consequence of proton-capture reactions occurring near the H-burning shell in some astrophysical source (as originally suggested in this context by Denisenkov \& Denisenkova 1990 and Langer et al. 1993). The reactions involved are those of the $\mathrm{Ne}-\mathrm{Na}$ and $\mathrm{Mg}$-Al cycles (e.g., Cavallo et al. 1996). Magnesium abundances, and in particular $\mathrm{Mg}$ isotope ratios, have played a crucial role in the development of theories for the origin of the abundance anomalies. In particular, the outcome of the pioneering work of Shetrone (1996) helped rule out individual star "deep mixing" scenarios as a possible explanation for the observed abundance differences. Specifically, Shetrone (1996) analyzed spectra of two O-rich and four extremely O-poor red giants in M13. The O-poor stars were also strongly Al-enhanced and notably Mg-depleted. The key result was the recognition that the Mg-depletions in the O-poor stars required depletions in the ${ }^{24} \mathrm{Mg}$ abundance, with the ${ }^{25} \mathrm{Mg}$ and ${ }^{26} \mathrm{Mg}$ isotopes having above solar abundance ratios. Since the $p$-capture reaction on ${ }^{24} \mathrm{Mg}$ in the $\mathrm{Mg}-\mathrm{Al}$ cycle is strongly temperature dependent, significant ${ }^{24} \mathrm{Mg}$-depletions occur only for temperatures well above those found near the H-burning shell in canonical low mass stellar models. Consequently, while evolutionary mixing does occur in red giant branch stars, the primary origin of the abundance anomalies is now accepted as the result of events occurring during the formation of globular clusters that involve the inclusion of material processed in either rapidly rotating massive stars (e.g., Decressin et al. 2007), massive binary stars (de Mink et al. 2009), or relatively massive asymptotic giant branch (AGB) stars (e.g., Ventura \& D'Antona 2005 ) into a second generation of cluster stars. Subsequent work by Yong et al. (2003a, 2006) on the Mg isotope ratios for red 
giants in the clusters NGC 6752 and M13, and by Meléndez \& Cohen (2009) for M71, have verified and extended the results of Shetrone (1996).

While there are extensive studies of the $\mathrm{O}-\mathrm{Na}$ anti-correlation in a number of clusters with different $[\mathrm{Fe} / \mathrm{H}]$ values (e.g., Carretta et al. 2009), the presence of an $[\mathrm{Fe} / \mathrm{H}]$ abundance range in $\omega$ Cen can be turned to advantage as it allows investigation of the $\mathrm{O}-\mathrm{Na}$ anti-correlation phenomenon as a function of $[\mathrm{Fe} / \mathrm{H}]$ in a single (admittedly complex) system. The richness of $\omega$ Cen also allows large samples of stars to be studied. The investigations of Johnson \& Pilachowski (2010) and Marino et al. (2011) show that the $\mathrm{O}-\mathrm{Na}$ correlation is present at all $[\mathrm{Fe} / \mathrm{H}]$ values except perhaps for the highest $[\mathrm{Fe} / \mathrm{H}]$ values $([\mathrm{Fe} / \mathrm{H}] \gtrsim-1.0 \mathrm{dex})$. In particular, the maximum value of $[\mathrm{Na} / \mathrm{Fe}]$ seen in O-depleted stars increases monotonically with $[\mathrm{Fe} / \mathrm{H}]$ reaching $[\mathrm{Na} / \mathrm{Fe}] \sim+1.0$ for the most metal-rich objects. In this latter population, Na-rich stars are dominant despite the apparent presence of a significant range in $[\mathrm{O} / \mathrm{Fe}]$ values. The situation for $[\mathrm{Al} / \mathrm{Fe}]$ is somewhat different-the maximum value of $[\mathrm{Al} / \mathrm{Fe}]$ in $\mathrm{O}$-poor, Na-rich stars is relatively constant at $[\mathrm{Al} / \mathrm{Fe}] \sim+1.2$ for stars with $[\mathrm{Fe} / \mathrm{H}] \lesssim-1.3$, but for higher metallicities the maximum value of $[\mathrm{Al} / \mathrm{Fe}]$ decreases significantly to $[\mathrm{Al} / \mathrm{Fe}] \sim+0.6$ at the highest metallicities (Norris \& Da Costa 1995b; Johnson \& Pilachowski 2010; Da Costa \& Marino 2011). Possible ways to generate these abundance patterns are discussed in, for example, Johnson \& Pilachowski (2010) and D'Antona et al. (2011).

The only extensive study of $\mathrm{Mg}$ in $\omega$ Cen stars, however, is that of Norris \& Da Costa (1995b). ${ }^{1}$ The results for the stars analyzed by these authors (whose data for $\mathrm{Na}, \mathrm{Al}$, and $\mathrm{O}$ exhibit the same trends with $[\mathrm{Fe} / \mathrm{H}]$ as those of Johnson \& Pilachowski 2010 and Marino et al. 2011) reveal only a minor population of stars with significant $\mathrm{Mg}$-depletions. The stars with $\mathrm{Mg}$-depletions are, as expected, strongly enhanced in $[\mathrm{Al} / \mathrm{Fe}]$ and generally also show significant depletions in $[\mathrm{O} / \mathrm{Fe}]$. In the terminology of Carretta et al. (2009), most belong to the extreme population. While the sample is not large compared to those of Johnson \& Pilachowski (2010) and Marino et al. (2011), it is notable that these $\mathrm{Mg}$-depleted stars are all relatively metal poor: the highest $[\mathrm{Fe} / \mathrm{H}]$ value is $-1.4 \mathrm{dex}$. This is no doubt related to the smaller $\mathrm{Al}$ enhancements seen at higher $[\mathrm{Fe} / \mathrm{H}]$ values (Norris \& Da Costa 1995b; Johnson \& Pilachowski 2010; Da Costa \& Marino 2011).

It is clear that further information on the process or processes underlying the $\mathrm{O}-\mathrm{Na}$ anti-correlation could be obtained by the measurement of $\mathrm{Mg}$ isotope ratios in a suitably selected set of $\omega$ Cen red giants. For example, in the $6 M_{\odot}$ metal-poor AGB model of Ventura et al. (2011), Mg-Al cycle burning at the bottom of the convective envelope produces surface $\mathrm{Mg}$ abundances that are dominated by ${ }^{25} \mathrm{Mg}$ with low ${ }^{26} \mathrm{Mg}$ abundances. This is in conflict with the existing data for Al-rich stars in NGC 6752 and M13 where ${ }^{26} \mathrm{Mg}$ has a higher abundance than ${ }^{25} \mathrm{Mg}$ (Yong et al. 2003a, 2006). Confirmation of this discrepancy would likely drive a reassessment of the theoretical treatments, such as mixing processes and nuclear reaction rates, employed in the stellar models.

Against this background, the purpose of the present paper is to determine the $\mathrm{Mg}$ isotope ratios for a representative set of red giants in $\omega$ Cen and for two red giants in the "normal" globular cluster M4 (NGC 6121). The selection of the sample

\footnotetext{
1 We note for completeness that Smith et al. (2000) derived $\mathrm{Mg}$ abundances for $10 \omega$ Cen red giants while Brown \& Wallerstein (1993) provided Mg abundances for six stars, one in common with Smith et al. (2000).
}

is described in Section 2, together with a presentation of the observations and data reduction. The analysis to derive the $\mathrm{Mg}$ isotope ratios is discussed in Section 3 and the results for the $\omega$ Cen and M4 stars are presented in Section 4. In Section 5, we combine our results with previous studies and discuss the implications in the context of existing models for the synthesis of the ${ }^{25} \mathrm{Mg}$ and ${ }^{26} \mathrm{Mg}$ isotopes.

\section{OBSERVATIONS AND REDUCTIONS}

\subsection{Sample Selection}

The $\omega$ Cen stars to be observed in this program were selected from the set of red giant members observed at high resolution and analyzed by Norris \& Da Costa (1995b). These authors give atmospheric parameters, iron abundances $[\mathrm{Fe} / \mathrm{H}]$, and elementto-iron abundance ratios for a biased sample of 40 stars that covers nearly the full range of $[\mathrm{Fe} / \mathrm{H}]$ values seen in the cluster. In particular, we first selected ROA 94, which with $[\mathrm{Fe} / \mathrm{H}]=$ -1.78 is one of the most metal-poor stars in $\omega$ Cen (Norris \& Da Costa 1995b; Johnson \& Pilachowski 2010; Marino et al. 2012). The star has an enhanced $[\alpha / \mathrm{Fe}]$ ratio and low values for $[\mathrm{Na} / \mathrm{Fe}],[\mathrm{Al} / \mathrm{Fe}]$, and [Ba/Fe] (Norris \& Da Costa 1995b). It is therefore a member of the primordial population of the cluster in the terminology of Carretta et al. (2009), and with the low $[\mathrm{Fe} / \mathrm{H}]$ value, a good candidate with which to characterize the "initial" value of the $\mathrm{Mg}$ isotope ratios in the cluster. The remaining stars in the observing list were pairs of stars selected to have similar $[\mathrm{Fe} / \mathrm{H}]$ values but with significantly different $[\mathrm{O} / \mathrm{Fe}],[\mathrm{Na} / \mathrm{Fe}]$, etc., abundances. In other words, we selected pairs of primordial and extreme population stars with similar $[\mathrm{Fe} / \mathrm{H}]$ values to investigate the difference in the $\mathrm{Mg}$ isotopic ratios between the different populations. The set of pairs also encompassed a range of $[\mathrm{Fe} / \mathrm{H}]$ values so that any difference between the populations as a function of $[\mathrm{Fe} / \mathrm{H}]$ could be explored. Three such pairs of stars were subsequently observed: ROA 43 and 100, ROA 132 and 150, and ROA 201 and 248. Their abundance characteristics and atmospheric parameters along with those for ROA 94 are given in Table 1. The values are taken from Norris \& Da Costa (1995b).

We note that the primordial stars ROA 43, ROA 132, and ROA 201 belong to the "CO-strong" population in $\omega \mathrm{Cen}$, while the extreme stars ROA 150 and ROA 248 belong to the "COweak" population. No CO-strength classification is available for ROA 94 or ROA 100. As discussed in Persson et al. (1980) and Norris \& Da Costa (1995b, Section 2.1), the $\omega$ Cen COstrong stars have stronger near-IR CO indices than those of all of the red giants in other globular clusters for which Persson et al. (1980) present data, while the CO-weak stars have CO indices comparable to those of other globular cluster red giants at similar $[\mathrm{Fe} / \mathrm{H}]$ values. For completeness, we note also that the extreme stars ROA 100 and ROA 150 are classified "CN-strong" in Norris \& Da Costa (1995b) while the primordial star ROA 94 is classified "CN-weak." No CN-band strength classification information is available for the primordial stars ROA 43, ROA 132, and ROA 201 and for the extreme star ROA 248.

Six of our seven stars have also been observed in Johnson \& Pilachowski (2010): stars ROA 94, 43, 100, 132, 150, and 248 correspond to Johnson \& Pilachowski (2010) stars 39037 , 10012, 44449, 55114, 73025, and 55071, respectively. For these six stars, we find mean $[\mathrm{Fe} / \mathrm{H}],[\mathrm{O} / \mathrm{Fe}]$, and $[\mathrm{Na} / \mathrm{Fe}]$ differences, in the sense Norris \& Da Costa (1995b)-Johnson \& Pilachowski (2010) of $0.00,-0.01$, and +0.03 dex, respectively. The standard deviations are $0.10,0.13$, and 0.09 dex, which 
Table 1

Globular Cluster Program Stars

\begin{tabular}{|c|c|c|c|c|c|c|c|c|c|}
\hline Object & $T_{\text {eff }}$ & $\log g$ & {$[\mathrm{Fe} / \mathrm{H}]$} & {$[\mathrm{C} / \mathrm{Fe}]$} & {$[\mathrm{O} / \mathrm{Fe}]$} & {$[\mathrm{Na} / \mathrm{Fe}]$} & {$[\mathrm{Mg} / \mathrm{Fe}]$} & {$[\mathrm{Al} / \mathrm{Fe}]$} & Pop Type \\
\hline$\omega$ Cen ROA 94 & 4200 & 0.7 & -1.78 & -0.67 & 0.25 & -0.26 & 0.51 & $<0.17$ & $\mathrm{P}$ \\
\hline$\omega$ Cen ROA 43 & 3950 & 0.4 & -1.47 & -0.21 & 0.39 & 0.21 & 0.52 & 0.06 & $\mathrm{P}$ \\
\hline$\omega$ Cen ROA 100 & 4150 & 0.7 & -1.49 & $<-0.54$ & $<-0.43$ & 0.58 & 0.05 & 1.15 & $\mathrm{E}$ \\
\hline$\omega$ Cen ROA 132 & 3900 & 0.3 & -1.37 & -0.22 & 0.35 & 0.17 & 0.56 & -0.32 & $\mathrm{P}$ \\
\hline$\omega$ Cen ROA 150 & 3950 & 0.6 & -1.25 & -0.83 & -0.46 & 0.59 & 0.26 & 1.06 & $\mathrm{E}$ \\
\hline$\omega$ Cen ROA 201 & 3750 & 0.5 & -0.85 & -0.30 & 0.37 & -0.12 & 0.45 & -0.07 & $\mathrm{P}$ \\
\hline$\omega$ Cen ROA 248 & 3850 & 0.6 & -0.78 & $\ldots$ & -0.33 & 0.75 & 0.24 & 0.68 & $\mathrm{E}$ \\
\hline NGC 6121 L1514 & 3875 & 0.3 & -1.18 & -1.00 & 0.41 & 0.01 & 0.40 & 0.44 & $\mathrm{P}$ \\
\hline NGC 6121 L2406 & 4100 & 0.5 & -1.18 & -0.84 & 0.19 & 0.31 & 0.37 & 0.55 & I \\
\hline NGC 6752 mg0 & 3928 & 0.3 & -1.62 & $\ldots$ & -0.08 & 0.65 & 0.46 & 1.06 & $\mathrm{E}$ \\
\hline
\end{tabular}

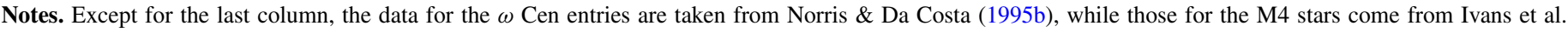

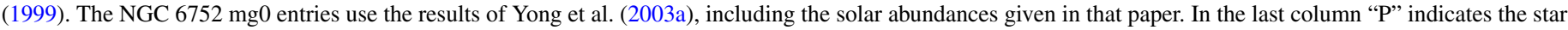

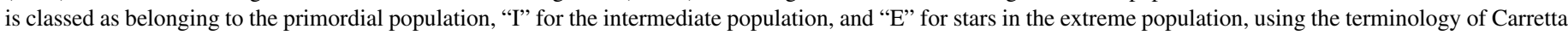

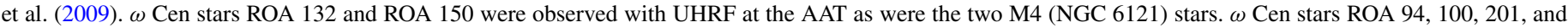
248, together with NGC $6752 \mathrm{mg0}$, were observed with b-HROS at Gemini-S. Star ROA 43 was observed at both facilities.

are consistent with the combined uncertainties in the abundance determinations. Although Johnson \& Pilachowski (2010) do not list $[\mathrm{Al} / \mathrm{Fe}]$ ratios for the six common stars, $[\mathrm{Al} / \mathrm{Fe}]$ values are given in Johnson et al. (2008). Using those data, we find a mean difference in $[\mathrm{Al} / \mathrm{Fe}]$ in the sense Norris \& Da Costa (1995b)-Johnson et al. (2008) of -0.08 dex though with a disturbingly large standard deviation of 0.47 dex. Since the $[\mathrm{Al} / \mathrm{Fe}]$ determinations of Norris \& Da Costa (1995b) are in good agreement with those in Smith et al. (2000; mean difference of -0.07 dex with $\sigma=0.16$ dex for three stars which cover an $[\mathrm{Al} / \mathrm{Fe}]$ range of $\sim 1 \mathrm{dex}$ ), we ascribe the large $\sigma$ primarily to large uncertainties in the Johnson et al. (2008) $[\mathrm{Al} / \mathrm{Fe}]$ determinations. In that context, it is worth noting that the Johnson et al. (2008) spectra have only moderate spectral resolution $R \sim 13,000$ as distinct from $R \sim 18,000$ for Johnson $\&$ Pilachowski (2010) and $R \sim 38,000$ for Norris \& Da Costa (1995b). Similarly, star ROA 132 is the only one from our sample in common with the work of Marino et al. (2011); it corresponds to their star 206284. The abundance differences in the sense Norris \& Da Costa (1995b)-Marino et al. (2011) are $0.19,-0.18$, and +0.07 dex for $[\mathrm{Fe} / \mathrm{H}],[\mathrm{O} / \mathrm{Fe}]$, and $[\mathrm{Na} / \mathrm{Fe}]$, respectively. Unfortunately, this star is not among those with $\mathrm{C}$, $\mathrm{N}$, and $\mathrm{O}$ abundance measures in Marino et al. (2012).

The two M4 stars were selected as the brightest pair of "CN-weak" (L1514) and "CN-strong" (L2406) stars in the detailed abundance study of M4 red giants carried out by Ivans et al. (1999). Based on the $[\mathrm{O} / \mathrm{Fe}]$ and $[\mathrm{Na} / \mathrm{Fe}]$ values given in Ivans et al. (1999), star L1514 is a member of the primordial population of M4 while L2406, which has an oxygen abundance 0.22 dex lower than L1514 and a sodium abundance 0.30 dex higher, is a member of the cluster's intermediate population. The two stars differ marginally in $[\mathrm{Al} / \mathrm{Fe}]$ with $\mathrm{L} 26140.11 \mathrm{dex}$ higher, but have essentially identical $\mathrm{Mg}$ abundances. Indeed in the sample of M4 red giants observed by Ivans et al. (1999), there is no evidence for any intrinsic range in $[\mathrm{Mg} / \mathrm{Fe}]$ or any obvious correlation between $[\mathrm{Mg} / \mathrm{Fe}]$ and $[\mathrm{Na} / \mathrm{Fe}]$ or $[\mathrm{Al} / \mathrm{Fe}]$ (Ivans et al. 1999). The atmospheric parameters and abundances for the two stars from Ivans et al. (1999) are given in Table 1. The two M4 stars were also included in the study of Yong et al. (2008b) who found similar results to Ivans et al. (1999). In particular, Yong et al. (2008b) find an oxygen abundance difference of 0.18 dex (in the sense L1514-L2406) and
$[\mathrm{Na} / \mathrm{Fe}],[\mathrm{Mg} / \mathrm{Fe}]$, and $[\mathrm{Al} / \mathrm{Fe}]$ differences of $-0.36,-0.08$, and $-0.03 \mathrm{dex}$, respectively. The oxygen and sodium abundances confirm the population classification for these stars.

Star L1514 is also included in the M4 study of Marino et al. (2008), but L2406 is not. Using the oxygen and sodium abundances of Marino et al. (2008), L1514 is again classified as a primordial star. As did Ivans et al. (1999) and Yong et al. (2008b), Marino et al. (2008) find no significant magnesium-aluminum anti-correlation, and that $[\mathrm{Mg} / \mathrm{Fe}]$ does not correlate with either $[\mathrm{O} / \mathrm{Fe}]$ or (anti-)correlate with $[\mathrm{Na} / \mathrm{Fe}]$ within their extensive sample of M4 red giant members. The total $[\mathrm{Al} / \mathrm{Fe}]$ range is also small at $\lesssim 0.4$ dex (Marino et al. 2008).

\subsection{Observations}

The observations were obtained with two different telescope/ spectrograph combinations. The first employed the "UltraHigh Resolution Facility" (UHRF) ${ }^{2}$ on the Anglo-Australian Telescope (AAT). The instrument was used in the resolution 300,000 mode but with a $0{ }^{\prime} .6$ slit in place of the normal imager slicer. This setup yields spectra with an effective resolution $R \sim 100,000$ and a scale of $0.018 \AA$ per binned pixel with the Tek $1 \mathrm{k} \times 1 \mathrm{k}$ detector in use at the time. Due to the limited wavelength coverage available with this detector, the central wavelength of the spectrograph setup had to be adjusted for the geocentric velocity of each target to ensure the isotopic abundance sensitive $\mathrm{MgH}$ features $(\lambda \sim 5134.55-5140.2 \AA)$ were centered. Data were obtained in two runs in 2000 April and 2001 March under seeing conditions that varied from $1^{\prime \prime} .2$ to 2 .'4, with a typical value of 1 ". 8 . As the small slit size compared to the seeing limits the instrument efficiency, total exposure times of many hours (broken into individual $1800 \mathrm{~s}$ integrations) were needed to achieve signal-to-noise ratio $(\mathrm{S} / \mathrm{N})$ values that would be useful for this project. In the event, as we shall see below, we were able to obtain $\mathrm{S} / \mathrm{N}$ values of $\sim 50-60$ per pixel for the $\omega$ Cen stars ROA 43, 132, and 150 with this instrument setup for total exposure time of 10, 10, and $17.5 \mathrm{hr}$, respectively. For the M4 stars L1514 and L2406, the S/N values were $\sim 70$ and $\sim 45$ for total exposure times of 2.0 and $3.5 \mathrm{hr}$, respectively. We also obtained spectra of bright field stars for which $\mathrm{Mg}$ isotope ratios have been determined by Gay \& Lambert (2000). Flat

\footnotetext{
2 www.aao.gov.au/astro/uhrf.html
} 
field, Th-Ar arc lamp, and dark frame exposures were obtained each night during both observing runs.

The second telescope/instrument combination used was the (now decommissioned) b-HROS ${ }^{3}$ bench-mounted fiber-fed high dispersion spectrograph on the Gemini-S $8 \mathrm{~m}$ telescope. The spectrograph was located in the pier of the telescope and fed via fibers from a cassette mounted in GMOS-S that locates them in the focal plane of the telescope. Guiding and tip/tilt image motion compensation is achieved through use of the GMOS-S On-Instrument Wavefront Sensor. We used the "object only" mode in which a single fiber with a projected diameter of $0{ }^{\prime} .9$ on the sky transfers the starlight to the spectrograph. An image-slicer at the spectrograph entrance slit then reformats the fiber output into seven $0{ }^{\prime}$. 14 wide slices with a nominal resolution of $R \sim 150,000$. The central wavelength of the echelle was set to $5135 \AA$ in order 40 . With the single $2048 \times$ 4603 pixel (spatial $\times$ wavelength) $\mathrm{CCD}$ detector this provided sufficient wavelength coverage that no adjustment in the central wavelength was required to compensate for the range of radial velocities exhibited by the various targets. On-chip binning of $\times 4$ in the spatial direction was used and the scale in the spectral direction was $0.010 \AA$ per pixel.

The observations were carried out during a two night (2006 UT March 16 and 18) classical run. Both nights were clear with excellent seeing $\left(\sim 0^{\prime \prime}\right.$. 6$)$. Typical total exposures for the program targets were of order $2 \mathrm{hr}$ per star, broken into individual $1800 \mathrm{~s}$ integrations. Observations were obtained of $\omega$ Cen red giants ROA 43, 94, 100, 201, and 248, where we note that ROA 43 was also observed with the AAT/UHRF setup. In addition, we also observed the globular cluster red giant NGC $6752 \mathrm{mg} 0$ which has been analyzed by Yong et al. (2003a), whose data we present for comparison purposes in the final row of Table 1. Spectra were also obtained of three bright field stars for which $\mathrm{Mg}$ isotope ratios have been determined by Gay \& Lambert (2000) or by Yong et al. (2003a). Flat field and arc lamp exposures were also obtained each night using the G-CAL facility calibration unit. All frames were bias-subtracted and trimmed of the overscan regions at the telescope.

\subsection{Data Reduction}

The data for the AAT/UHRF and Gemini-S/b-HROS configurations were reduced by using the FIGARO package. ${ }^{4}$ Individual spectra were flat-fielded and wavelength calibrated with spectra of the quartz and arc lamps, respectively; they were cosmic-ray cleaned using "bclean"; curvature in the spectra was removed with "sdist" and "cdist"; individual observations of each object were wavelength shifted to bring them into the rest frame; and for each object the individual spectra were co-added, sky-subtracted, and finally continuum normalized by using spectra of a rapidly rotating $\mathrm{B}$ star ( $\beta$ Centauri) to constrain the shape of the instrumental response function. An essential difference between the two instrumental configurations is that for UHRF the spectral lines were set perpendicular to the dispersion direction on the detector, while for b-HROS this was not the case. For the latter, we used curvature-corrected arc spectra to determine and remove the slope of the spectral lines in the spatial direction by estimating the differential wavelength shifts in that direction, and shifting the stellar spectra by the appropriate amounts before summing the counts.

\footnotetext{
3 www.gemini.edu/sciops/instruments/hros/hrosindex.html accessible via www.gemini.edu/node/10002\#obsolete

4 www.aao.gov.au/figaro
}

For the three $\omega$ Cen stars observed at the AAT with UHRF, the net counts in the continuum near $5140 \AA$ lie in the range 2700-3100 electrons per $0.018 \AA$ pixel. For M4 L1514 and L2406, the net counts were 4800 and 2200 electrons per pixel, respectively. This suggests that the $\mathrm{S} / \mathrm{N}$ values for these AAT globular cluster red giant spectra lie in the range $\sim 50-70$ per pixel. For the Gemini-S data, the net counts range from 6300 (ROA 43) to 15,900 (NGC $6752 \mathrm{mg} 0$ ) electrons per $0.010 \AA$ pixel, suggesting $\mathrm{S} / \mathrm{N}$ in the range $\sim 80-130$ per pixel. These values are confirmed by a direct determination of the rms dispersion of the counts in the stellar continuum in small bands of width $\sim 0.3 \AA$ at $5135.5 \AA$ and $5137.9 \AA$ in the spectra of the hotter and higher $\mathrm{S} / \mathrm{N} \omega$ Cen stars ROA 94 and 100 and in NGC $6752 \mathrm{mg} 0$. The rms dispersions lead to $\mathrm{S} / \mathrm{N}$ values in the range 90-130, consistent with those calculated from the net counts.

Figures 1 and 2 show the final reduced spectra for the $\omega$ Cen stars over wavelength regions that include electronic transitions of the $\mathrm{MgH}$ molecule, and the features specific to each $\mathrm{Mg}$ isotope are identified. Similarly, Figure 3 shows the isotopic ratio sensitive regions for the two M4 stars. Apparent differences in the isotopic compositions are immediately evident from these figures, especially for the $\omega$ Cen stars. We now seek to quantify the differences.

\section{ABUNDANCE ANALYSIS}

The isotopic abundances of magnesium were measured using the identical approach to that in Yong et al. (2003a, 2004, 2006) and thus can be briefly described here. We used one-dimensional local thermodynamic equilibrium (LTE) model atmospheres from Kurucz (1996) and adopted stellar parameters for the $\omega$ Cen stars from Norris \& Da Costa (1995b), from Ivans et al. (1999) for the M4 stars and from Yong et al. (2003a) for NGC 6752 mg0. The particular stellar parameters employed are not crucial as Yong et al. (2004) have shown that the derived isotope ratios appear insensitive to the adopted stellar parameters, nonLTE effects, and inadequacies in the model atmospheres. We analyzed the three lines from the $A-X$ electronic transition of the $\mathrm{MgH}$ molecule recommended by McWilliam \& Lambert (1988) as providing the most reliable isotope ratios (the numerous other $\mathrm{MgH}$ lines present in the spectra are more strongly affected by known, and unknown, blends). The three lines are (1) $5134.6 \AA$ from the $Q_{1}(23)$ and $R_{2}(11)$ lines of the $(0,0)$ band, (2) $5138.7 \AA$ from the $(0,0) Q_{1}(22)$ and $(1,1) Q_{2}(14)$ lines, and (3) $5140.2 \AA$ from the $(0,0) R_{1}(10)$ and $(1,1) R_{2}(4)$ lines.

We note that other authors choose to analyze different $\mathrm{MgH}$ features-for example, Meléndez \& Cohen (2009) prefer the $5134.3 \AA$ feature to that at $5134.6 \AA$-potentially resulting in systematically different isotopic ratios. We therefore make no strong claim for the validity of the absolute values of our isotopic ratios but argue that consistent analysis allows meaningful comparisons in a relative sense. It is also likely that known and unknown blends affecting the $\mathrm{MgH}$ analysis are more problematic for cooler and more metal-rich stars, potentially leading to feature-to-feature differences in the derived isotopic ratios. Non-LTE and three-dimensional effects may also become more important for cooler temperatures. Some care must then be taken in comparing results as a function of metallicity although as shown below, our results are consistent with published values. For example, our $\mathrm{Mg}$ isotopic abundance determination for the cool red giant HR $2140\left(T_{\text {eff }}=4000 \mathrm{~K}, \log g=0.9,[\mathrm{Fe} / \mathrm{H}]=\right.$ -0.9 ; McWilliam \& Lambert 1988) is consistent with that of McWilliam \& Lambert (1988). Indeed, the comparison of our 


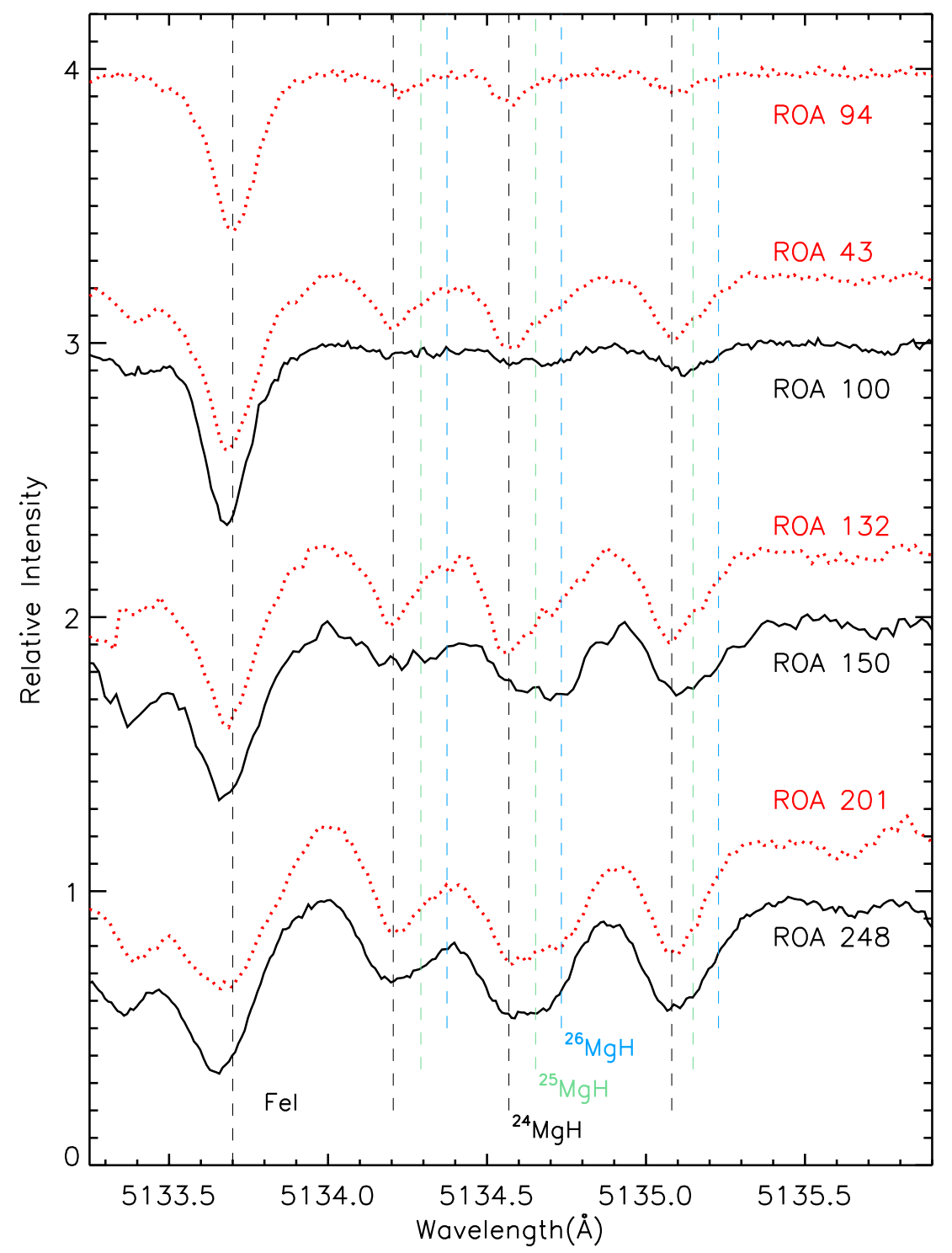

Figure 1. Spectra for the $\omega$ Cen program stars for wavelengths between $5133 \AA$ and $5136 \AA$. The spectra are ordered approximately by metallicity with ROA 94 being the most metal poor and ROA 248 the most metal rich, respectively. The primordial population star spectra are plotted as red dots, while those for the extreme population stars are plotted as black lines. The positions of the ${ }^{24} \mathrm{MgH},{ }^{25} \mathrm{MgH}$, and ${ }^{26} \mathrm{MgH}$ features are marked by vertical lines.

(A color version of this figure is available in the online journal.)

results with published values that use the same MgH wavelength regions argues that our results are internally consistent, although a relative offset cannot be ruled out. Nevertheless, additional studies seeking to identify blends affecting these and other $\mathrm{MgH}$ features would be welcomed.

Synthetic spectra were generated using the LTE stellar line analysis program MOOG (Sneden 1973), with the line broadening, assumed to have a Gaussian form, estimated for each star by fitting the profile of the $5115.4 \AA \mathrm{Ni}$ I line. The values of the line broadening employed range from 6 to $10 \mathrm{~km} \mathrm{~s}^{-1}$, with an uncertainty of order $1-2 \mathrm{~km} \mathrm{~s}^{-1}$. Each of the three $\mathrm{MgH}$ lines was analyzed independently with three free parameters: the ${ }^{25} \mathrm{Mg} /{ }^{24} \mathrm{Mg}$ and ${ }^{26} \mathrm{Mg} /{ }^{24} \mathrm{Mg}$ isotopic ratios and the total $\mathrm{Mg}$ abundance, $\log \epsilon(\mathrm{Mg})$. A large range of parameter space was explored and the optimum value for each parameter identified by locating the $\chi^{2}$ minima. Following Bevington \& Robinson (1992), the $1 \sigma$ confidence limit for a given parameter was taken as the value at which $\Delta \chi^{2}=\chi^{2}-\chi_{\min }^{2}=1$. Thus, for each line in each star, we obtain an optimum value and an uncertainty for
${ }^{25} \mathrm{Mg} /{ }^{24} \mathrm{Mg}$ and ${ }^{26} \mathrm{Mg} /{ }^{24} \mathrm{Mg}$. The final ratio ${ }^{24} \mathrm{Mg}:{ }^{25} \mathrm{Mg}:{ }^{26} \mathrm{Mg}$ was then calculated as the weighted mean of the three determinations. The individual determinations and the final adopted values for the $\omega$ Cen and M4 stars, and NGC $6752 \mathrm{mg} 0$, are given in Table 2.

When expressing the isotopic ratios as ${ }^{24} \mathrm{Mg}:{ }^{25} \mathrm{Mg}:{ }^{26} \mathrm{Mg}=$ $(100-b-c): b: c$, the formal errors in the determinations from each region are small; $b \pm 1$ and $c \pm 1$. However, inspection of the values in Table 2, for example, suggests that the real uncertainties are larger, being driven by systematic uncertainties in the continuum fitting, the adopted macroturbulent velocities, and (known and unknown) blends. As discussed in previous studies of stellar $\mathrm{Mg}$ isotope ratios, the ${ }^{25} \mathrm{Mg} /{ }^{24} \mathrm{Mg}$ ratio is often more uncertain than the ${ }^{26} \mathrm{Mg} /{ }^{24} \mathrm{Mg}$ ratio since the isotopic shift is smaller, and as a result, the ${ }^{25} \mathrm{MgH}$ feature is less well separated from the (generally) dominant ${ }^{24} \mathrm{MgH}$ line. This effect can be seen qualitatively in what follows in Figures 5-7 and quantitatively from the $\chi^{2}$ analysis. We also note that the isotope ratios derived from each of the three regions are usually, but not 


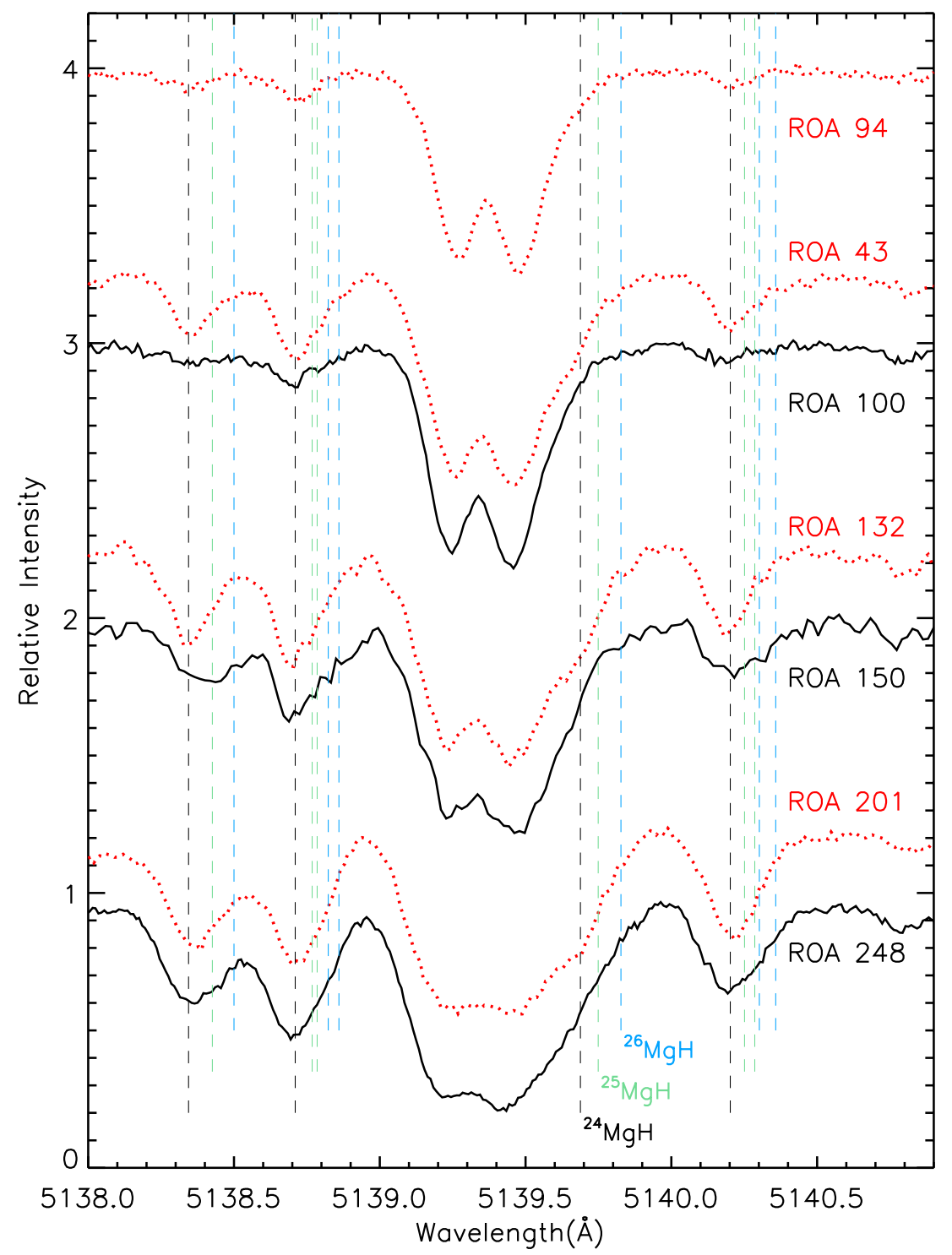

Figure 2. Same as for Figure 1 except that the wavelength region shown is 5138-5141 A.

(A color version of this figure is available in the online journal.)

always, in good agreement. In some rare cases (e.g., $\omega$ Cen ROA 201 ), the ratios from the three regions show poor agreement for which we have no obvious explanation.

An additional assessment of the uncertainties can be obtained from independent analysis of repeat observations, and from a comparison of isotopic ratios found here with the results of others. For example, three separate spectra of Arcturus, obtained during the AAT/UHRF runs, give ${ }^{24} \mathrm{Mg}:{ }^{25} \mathrm{Mg}:{ }^{26} \mathrm{Mg}$ ratios of 81:9:10, 84:6:10, and 80:10:10 which are all in good agreement with the value 82:9:9 found by McWilliam \& Lambert (1988). There are also three separate AAT/UHRF spectra of the star HR 2140. As for Arcturus, our determinations for this star of 80:10:10, 82:8:10, and 83:7:10 are in excellent accord with each other and with the value 88:6:6 given in McWilliam \& Lambert (1988). Similarly, during the Gemini-S/b-HROS run three field stars with previous Mg isotope ratio determinations were observed. The stars are HD 26575 (HR 1299), HD 64606, and HD 103036 and we find isotopic ratios of $81: 5: 14,89: 2: 9$, and 87:2:11, respectively. The published isotopic ratio determinations for the first two are 72:18:10 and 82:10:8, respectively, from Gay \& Lambert (2000), while
Yong et al. (2003a) list 94:0:6 for HD 103036. Our values are consistent with the previous determinations with the possible exception of the ${ }^{25} \mathrm{Mg} /{ }^{24} \mathrm{Mg}$ ratio for the $\sim$ solar abundance star HD 26575 where the Gay \& Lambert (2000) value ${ }^{5}$ is notably higher than the value from our analysis. We note, however, that our isotopic ratios for this star are consistent with the 80:10:10 determination given by McWilliam \& Lambert (1988). Perhaps more significantly, we find from our GeminiS/b-HROS observations an isotope ratio of 62:9:29 for the star NGC $6752 \mathrm{mg} 0$, which is in excellent agreement with the value of 63:8:30 given in Yong et al. (2003a) from VLT/UVES spectra. The isotopic ratios derived from the three wavelength regions and the mean values for this star are also given in Table 2 . Overall, based on these comparisons, we estimate that the uncertainties in our determinations are at the level $b \pm 4$ and $c \pm 4$ when writing the ratio as ${ }^{24} \mathrm{Mg}:{ }^{25} \mathrm{Mg}:{ }^{26} \mathrm{Mg}=(100-$ $b-c): b: c$.

\footnotetext{
5 The value of ${ }^{25} \mathrm{Mg} /{ }^{24} \mathrm{Mg}$ for $\mathrm{HD} 26575(0.25)$ is not shown in the ${ }^{25} \mathrm{Mg} /{ }^{24} \mathrm{Mg}$ versus $[\mathrm{Fe} / \mathrm{H}]$ diagram of Gay \& Lambert (2000, their Figure 8) as it lies outside the range depicted, notably higher than the ${ }^{25} \mathrm{Mg} /{ }^{24} \mathrm{Mg}$ values for stars of comparable metallicity.
} 

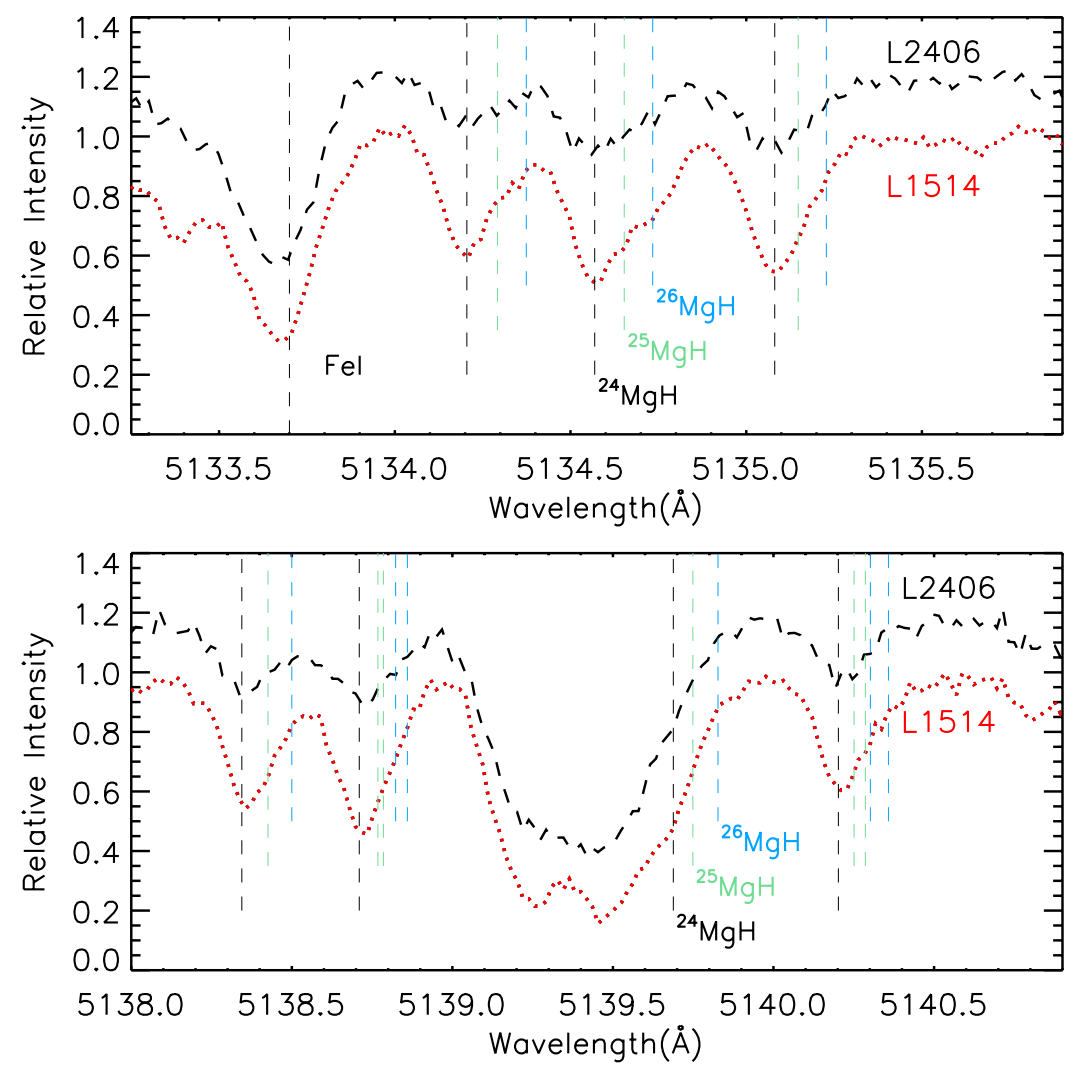

Figure 3. Upper panel shows the wavelength region 5133-5136 ̊n and the lower panel shows the wavelength region 5138-5141 ̊ for the M4 primordial population star L1514 (red dots) and for the M4 intermediate population star L2604 (black dashed line). As in Figures 1 and 2, the positions of the ${ }^{24} \mathrm{MgH},{ }^{25} \mathrm{MgH}$, and ${ }^{26} \mathrm{MgH}$ lines are marked by vertical lines.

(A color version of this figure is available in the online journal.)

\section{RESULTS}

In the following, we will make use of the results of Yong et al. (2003a) for the red giants in NGC 6752 and of Yong et al. (2006) for M13 and M71, which have been derived using essentially the same procedure as that adopted here. For NGC 6752, using the terminology of Carretta et al. (2009) and the sodium and oxygen abundances given in Yong et al. (2003a), stars NGC $6752 \mathrm{mg} 12$ and NGC $6752 \mathrm{mg} 24$ are classified as members of the primordial population, while stars NGC $6752 \mathrm{mg} 0, \mathrm{mg} 21, \mathrm{mg} 22$, and 702 are members of the extreme population. The other 14 red giants are members of the intermediate population. Similarly, for the four M13 red giants studied in Yong et al. (2006), and again using the sodium and oxygen abundances from that work, star L598 is a member of the primordial population, star L629 is a member of the intermediate population and stars L70 and L973 both belong to the extreme population. These four stars are a subset of those studied by Shetrone (1996). Yong et al. (2006) separate ${ }^{25} \mathrm{Mg}$ from ${ }^{26} \mathrm{Mg}$ but both studies find similar $\left({ }^{25} \mathrm{Mg}+{ }^{26} \mathrm{Mg}\right) /$ ${ }^{24} \mathrm{Mg}$ ratios for a given star. Further, the single M71 star also analyzed in Yong et al. (2006) is a CN-weak object (Meléndez \& Cohen 2009) with sodium and oxygen abundances (Yong et al. 2006; Meléndez \& Cohen 2009) that classify it as a member of the primordial population of the cluster. The sodium-to-iron, magnesium-to-iron, and aluminum-to-iron abundance ratios as a function of $[\mathrm{O} / \mathrm{Fe}]$ for all these stars are shown in Figure 4, along with the equivalent data for the $\omega$ Cen and M4 stars from Table 1.

\section{1. $\omega$ Cen ROA 94}

For this metal-poor star, we find ${ }^{24} \mathrm{Mg}:{ }^{25} \mathrm{Mg}:{ }^{26} \mathrm{Mg}$ values of $88: 2: 9$ with the standard uncertainty in the ratios of $b \pm 4$ and $c \pm 4$ where ${ }^{24} \mathrm{Mg}:{ }^{25} \mathrm{Mg}:{ }^{26} \mathrm{Mg}=(100-b-c): b: c$. The relative abundances of ${ }^{25} \mathrm{Mg}$ and ${ }^{26} \mathrm{Mg}$ found here for this star are somewhat higher, particularly for ${ }^{26} \mathrm{Mg}$, than those for field halo dwarfs at this metallicity, which are typically 97:1:2 (e.g., Meléndez \& Cohen 2007). However, the relative Mg isotope abundances appear to be somewhat lower than the $\sim 80: 10: 10$ values found for the primordial red giants in NGC 6752 and M13 (Yong et al. 2003a, 2006). The $[\mathrm{Fe} / \mathrm{H}]$ values for these clusters are higher, by $\sim 0.2$ dex, than that of ROA 94 .

\section{2. $\omega$ Cen ROA $43 / 100$ and ROA $132 / 150$}

As the derived $\mathrm{Mg}$ isotope ratios for these two pairs of stars are found to be similar (see Table 2), we discuss them together. Figures 5 and 6 show our fits to the $5134.6 \AA \mathrm{MgH}$ feature; the fits to the $5138.7 \AA$ and $5140.2 \AA \mathrm{MgH}$ features are similar. For the primordial stars ROA 43 and ROA 132, we find essentially identical relative abundances of ${ }^{24} \mathrm{Mg}:{ }^{25} \mathrm{Mg}:{ }^{26} \mathrm{Mg}=76: 10: 14$, with again uncertainties of order \pm 4 in the $b$ and $c$ values. These values are in reasonable agreement with the $\sim 80: 10: 10$ values found for the primordial stars in NGC 6752 and M13 by Yong et al. (2003a, 2006). This is perhaps not surprising given that the $[\mathrm{Fe} / \mathrm{H}]$ values for NGC 6752 and M13 are only $\sim 0.1$ to $0.2 \mathrm{dex}$ lower than the $[\mathrm{Fe} / \mathrm{H}]$ values of the $\omega$ Cen stars, which are given in Table 1.

For the two extreme population stars, we find ${ }^{24} \mathrm{Mg}:{ }^{25} \mathrm{Mg}$ : ${ }^{26} \mathrm{Mg}=48: 13: 39$ for ROA 100 and ${ }^{24} \mathrm{Mg}:{ }^{25} \mathrm{Mg}:{ }^{26} \mathrm{Mg}=$ 54:17:30 for ROA 150. Here, given the larger contribution to the features from the heavier isotopes and the lower overall $\mathrm{Mg}$ abundance (see Table 1), the uncertainties in the $b$ and $c$ values are somewhat larger, of order \pm 10 . Nevertheless, the require- 


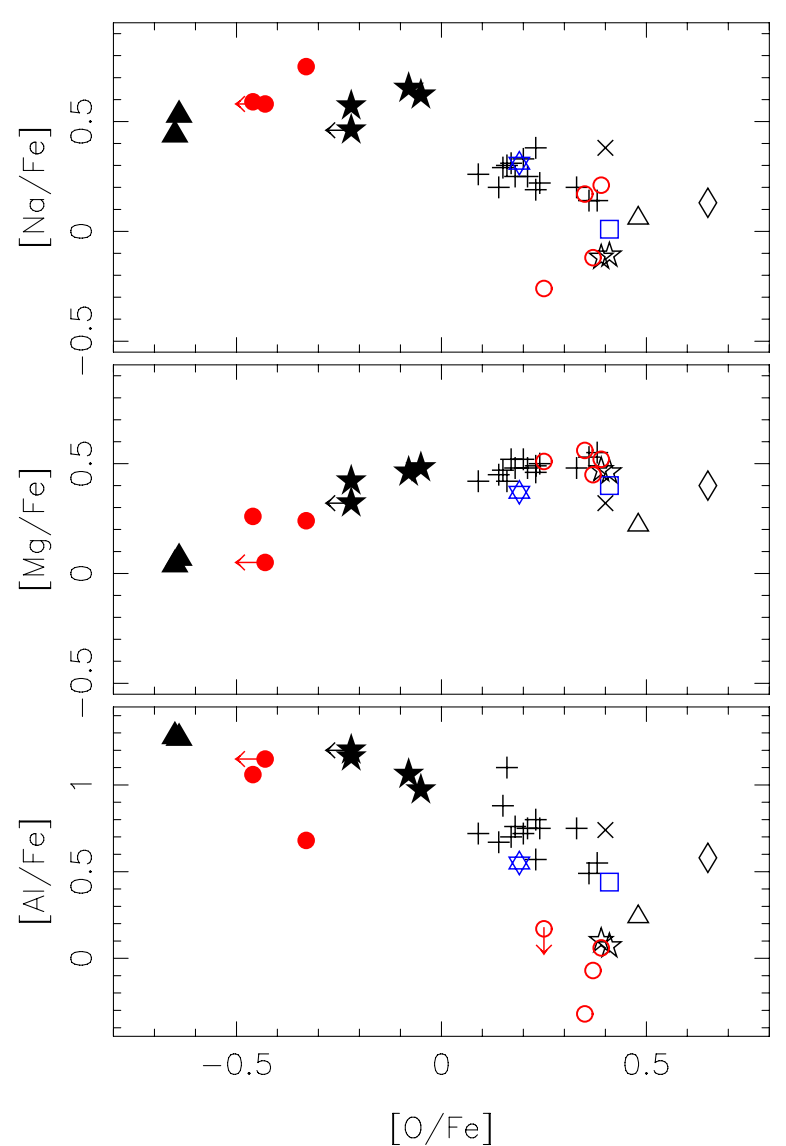

Figure 4. Sodium-to-iron (upper panel), magnesium-to-iron (middle panel), and aluminum-to-iron (lower panel) abundance ratios are plotted against the oxygen-to-iron abundance ratio. The three extreme population stars in $\omega$ Cen are shown as red filled circles while the four primordial population red giants are plotted as red open circles. The abundance ratios are taken from Norris \& Da Costa (1995b). The M4 stars L1514 (primordial population, blue square) and L2406 (intermediate population, blue six-pointed star) are plotted using the data of Ivans et al. (1999). The NGC 6752 data of Yong et al. (2003a) are plotted as filled stars (extreme population), open five-point stars (primordial population), and plus signs (intermediate population). Similarly, using the data from Yong et al. (2006), the two extreme population stars in M13 are plotted as filled triangles, the single primordial population star is shown as an open triangle, and the intermediate population star is plotted as a " $\times$ "symbol. The sole M71 star analyzed in Yong et al. (2006), which is a primordial population star, is plotted as an open diamond.

(A color version of this figure is available in the online journal.)

ment for significant contributions from the heavier isotopes to permit a good fit to the observations is readily apparent in the figures. The isotope ratios for these $\omega$ Cen extreme population stars are also similar to those found for the extreme populations in the clusters NGC 6752 and M13 (Yong et al. 2003a, 2006), namely, 62:7:31 for NGC 6752 (average of four stars) and 51:14:35 for M13 (average of two stars). Again the iron abundances for these two clusters are only $\sim 0.1-0.3$ dex lower than those of ROA 100 and ROA 150 (see Table 1).

In both pairs, the primary difference between the primordial and extreme stars is a decrease in the ${ }^{24} \mathrm{Mg}$ relative abundance and an increase in the ${ }^{26} \mathrm{Mg}$ relative abundance; the increase in the ${ }^{25} \mathrm{Mg}$ relative abundance appears more modest. We note also that the difference in total magnesium abundance between the primordial and extreme stars in each pair, derived as part of the fitting process, is consistent at the $0.1-0.15$ dex level with the differences inferred from Table 1. As argued by Yong et al. (2004), the total $\mathrm{Mg}$ abundances derived from the fits to the $\mathrm{MgH}$ features are not as reliable as those determined from individual $\mathrm{Mg}$ I lines; nevertheless, the overall agreement is reassuring.

\section{3. $\omega$ Cen ROA $201 / 248$}

For the O-rich, Na-poor primordial population star $\omega$ Cen ROA $201([\mathrm{Fe} / \mathrm{H}]=-0.85)$, our analysis yields ${ }^{24} \mathrm{Mg}:{ }^{25} \mathrm{Mg}:{ }^{26} \mathrm{Mg}=74: 19: 7$ while for the O-poor, Na-rich extreme population star $\omega$ Cen ROA $248([\mathrm{Fe} / \mathrm{H}]=-0.78)$ the corresponding values are 77:10:13. Given that the uncertainties in the derived ratios are somewhat larger for these cool relatively metal-rich stars, it is not clear whether we are seeing any significant difference in isotopic ratios. The synthetic spectrum fits to the $5134.6 \AA \mathrm{MgH}$ feature in the observed spectra are shown in Figure 7.

The lack of obvious difference in $\mathrm{Mg}$ isotopic composition between these two $\omega$ Cen stars is consistent with the results of Meléndez \& Cohen (2009) who found only a small difference in $\mathrm{Mg}$ isotopic ratios between the $\mathrm{CN}$-weak (primordial) and $\mathrm{CN}$ strong (intermediate) populations in the relatively metal-rich cluster M71. For example, the ${ }^{26} \mathrm{Mg} /{ }^{24} \mathrm{Mg}$ isotopic ratio for the $\mathrm{CN}$-strong stars exceeds that for the $\mathrm{CN}$-weak stars by only $\sim 4 \%$ (Meléndez \& Cohen 2009). The two M71 populations in their sample also do not show any significant difference as regards total $\mathrm{Mg}$ abundance, and the ranges in $\mathrm{O}, \mathrm{Na}$, and $\mathrm{Al}$ abundances are small although they do exhibit the expected $\mathrm{O}-\mathrm{Na}$ anticorrelation and $\mathrm{Na}-\mathrm{Al}$ correlation (Meléndez \& Cohen 2009). The $[\mathrm{Fe} / \mathrm{H}]$ value for M71 (-0.91; Yong et al. 2006), which we adopt here, is very similar to those for the $\omega$ Cen stars. Meléndez \& Cohen (2009) give $[\mathrm{Fe} / \mathrm{H}]=-0.80$ for M71.

\subsection{M4 L1514/L2406}

Figure 8 shows the spectrum synthesis fits to the $5134.6 \AA$ $\mathrm{MgH}$ feature for the two M4 stars. The fits for the three wavelength regions yield mean isotopic ratios of 82:6:12 for the primordial star L1514 and 80:6:15 for the intermediate population star L2406. Given the uncertainties in the determinations, there is no apparent difference in the derived $\mathrm{Mg}$ isotope ratios. Yong et al. (2008a) derived preliminary $\mathrm{Mg}$ isotope ratios for a number of M4 red giants. Because their spectra had a resolution of $\sim 60,000$, they assumed (as did Shetrone 1996) that ${ }^{25} \mathrm{Mg}=$ ${ }^{26} \mathrm{Mg}$. For L1514, they found an isotope ratio of 80:10:10, which is consistent with our result. An isotope ratio was not derived for L2406 but overall Yong et al. (2008a) did not find any significant evidence for a range in the $\mathrm{Mg}$ isotope ratios of their M4 stars.

The lack of any significant $\mathrm{Mg}$ isotope ratio difference for the two M4 stars observed here is perhaps not surprising given the lack of any difference in $[\mathrm{Mg} / \mathrm{Fe}]$ and the (at most) small difference in $[\mathrm{Al} / \mathrm{Fe}]$ for these stars (Ivans et al. 1999; Yong et al. 2008b). Again this is consistent with the emerging general picture that while the $\mathrm{O}-\mathrm{Na}$ anti-correlation is present at all metallicities, the extent of $\mathrm{Al}$ and especially $\mathrm{Mg}$ variations are much reduced at higher metallicities (e.g., Norris \& Da Costa 1995b; Ivans et al. 1999; Marino et al. 2008; Yong et al. 2008a; Meléndez \& Cohen 2009; Johnson \& Pilachowski 2010; Da Costa \& Marino 2011; Carretta et al. 2013).

\section{DISCUSSION}

As discussed in Kobayashi et al. (2011, see also Woosley \& Weaver 1995; Timmes et al. 1995), the heavy isotopes of magnesium have their origin in a number of nucleosynthetic processes. For example, explosive nucleosynthesis in massive 
Table 2

Globular Cluster Red Giant Mg Isotope Ratios

\begin{tabular}{|c|c|c|c|c|c|c|}
\hline Star & Region & ${ }^{25} \mathrm{Mg} /{ }^{24} \mathrm{Mg}$ & $\sigma$ & ${ }^{26} \mathrm{Mg} /{ }^{24} \mathrm{Mg}$ & $\sigma$ & ${ }^{24} \mathrm{Mg}:{ }^{25} \mathrm{Mg}:{ }^{26} \mathrm{Mg}$ \\
\hline \multirow[t]{4}{*}{$\omega$ Cen ROA 94} & $5134.6 \AA$ & 0.000 & 0.031 & 0.190 & 0.023 & $84: 00: 16$ \\
\hline & $5138.7 \AA$ & 0.006 & 0.054 & 0.029 & 0.038 & $97: 01: 03$ \\
\hline & $5140.2 \AA$ & 0.139 & 0.091 & 0.000 & 0.066 & $88: 12: 00$ \\
\hline & Mean & 0.027 & 0.016 & 0.106 & 0.012 & 88:02:09 \\
\hline \multirow[t]{4}{*}{$\omega$ Cen ROA 43} & $5134.6 \AA$ & 0.161 & 0.015 & 0.225 & 0.010 & $72: 12: 16$ \\
\hline & $5138.7 \AA$ & 0.167 & 0.021 & 0.148 & 0.011 & $76: 13: 11$ \\
\hline & $5140.2 \AA$ & 0.066 & 0.023 & 0.194 & 0.016 & $79: 05: 15$ \\
\hline & Mean & 0.137 & 0.006 & 0.190 & 0.004 & $75: 10: 14$ \\
\hline \multirow[t]{4}{*}{$\omega$ Cen ROA 100} & $5134.6 \AA$ & 0.368 & 0.068 & 1.005 & 0.065 & $42: 16: 42$ \\
\hline & $5138.7 \AA$ & 0.011 & 0.086 & 1.087 & 0.097 & $48: 01: 52$ \\
\hline & $5140.2 \AA$ & 0.497 & 0.115 & 0.461 & 0.072 & $51: 25: 24$ \\
\hline & Mean & 0.282 & 0.029 & 0.835 & 0.025 & $47: 13: 39$ \\
\hline \multirow[t]{4}{*}{$\omega$ Cen ROA 132} & $5134.6 \AA$ & 0.148 & 0.012 & 0.279 & 0.010 & $70: 10: 20$ \\
\hline & $5138.7 \AA$ & 0.064 & 0.017 & 0.204 & 0.011 & $79: 05: 16$ \\
\hline & $5140.2 \AA$ & 0.176 & 0.020 & 0.061 & 0.012 & $81: 14: 05$ \\
\hline & Mean & 0.130 & 0.005 & 0.188 & 0.004 & $76: 10: 14$ \\
\hline \multirow[t]{4}{*}{$\omega$ Cen ROA 150} & $5134.6 \AA$ & 0.908 & 0.091 & 2.189 & 0.106 & $24: 22: 53$ \\
\hline & $5138.7 \AA$ & 0.124 & 0.042 & 0.408 & 0.031 & $65: 08: 27$ \\
\hline & $5140.2 \AA$ & 0.203 & 0.053 & 0.234 & 0.029 & $70: 14: 16$ \\
\hline & Mean & 0.312 & 0.019 & 0.550 & 0.013 & $54: 17: 30$ \\
\hline \multirow[t]{4}{*}{$\omega$ Cen ROA 201} & $5134.6 \AA$ & 0.163 & 0.013 & 1.095 & 0.036 & $44: 07: 48$ \\
\hline & $5138.7 \AA$ & 0.278 & 0.025 & 0.036 & 0.004 & $76: 21: 03$ \\
\hline & $5140.2 \AA$ & 0.497 & 0.036 & 0.000 & 0.008 & $67: 33: 00$ \\
\hline & Mean & 0.259 & 0.007 & 0.098 & 0.002 & $74: 19: 07$ \\
\hline \multirow[t]{4}{*}{$\omega$ Cen ROA 248} & $5134.6 \AA$ & 0.165 & 0.014 & 0.481 & 0.017 & $61: 10: 29$ \\
\hline & $5138.7 \AA$ & 0.078 & 0.009 & 0.082 & 0.004 & $86: 07: 07$ \\
\hline & $5140.2 \AA$ & 0.241 & 0.027 & 0.230 & 0.012 & $68: 16: 16$ \\
\hline & Mean & 0.134 & 0.005 & 0.173 & 0.003 & $77: 10: 13$ \\
\hline \multirow[t]{4}{*}{ M4 L1514 } & $5134.6 \AA$ & 0.058 & 0.007 & 0.226 & 0.008 & $78: 05: 18$ \\
\hline & $5138.7 \AA$ & 0.111 & 0.014 & 0.062 & 0.010 & $85: 09: 05$ \\
\hline & $5140.2 \AA$ & 0.085 & 0.013 & 0.109 & 0.014 & 84:07:09 \\
\hline & Mean & 0.078 & 0.003 & 0.142 & 0.003 & $82: 06: 12$ \\
\hline \multirow[t]{4}{*}{ M4 L2406 } & $5134.6 \AA$ & 0.054 & 0.022 & 0.226 & 0.015 & $78: 04: 18$ \\
\hline & $5138.7 \AA$ & 0.053 & 0.038 & 0.243 & 0.025 & $77: 04: 19$ \\
\hline & $5140.2 \AA$ & 0.129 & 0.040 & 0.073 & 0.022 & $83: 11: 06$ \\
\hline & Mean & 0.073 & 0.010 & 0.185 & 0.007 & $80: 06: 15$ \\
\hline \multirow[t]{4}{*}{ NGC 6752 mg0 } & $5134.6 \AA$ & 0.163 & 0.019 & 0.495 & 0.014 & $60: 10: 30$ \\
\hline & $5138.7 \AA$ & 0.029 & 0.027 & 0.535 & 0.019 & $64: 02: 34$ \\
\hline & $5140.2 \AA$ & 0.285 & 0.038 & 0.339 & 0.023 & $62: 18: 21$ \\
\hline & Mean & 0.148 & 0.009 & 0.467 & 0.006 & $62: 09: 29$ \\
\hline
\end{tabular}

star supernovae can produce ${ }^{25} \mathrm{Mg}$ and ${ }^{26} \mathrm{Mg}$ through the He-burning reactions ${ }^{22} \mathrm{Ne}(\alpha, n)^{25} \mathrm{Mg}$ and ${ }^{22} \mathrm{Ne}(\alpha, \gamma)^{26} \mathrm{Mg}$. Being "secondary" isotopes, the production of the neutronrich isotopes increases with the metallicity of the supernovae, although some primary production also occurs (Kobayashi et al. 2011). The nucleosynthetic model predictions (Kobayashi et al. 2011) are at least qualitatively consistent with the observations of the variation of the $\mathrm{Mg}$ isotopic abundance ratios with $[\mathrm{Fe} / \mathrm{H}]$ in the field star samples of Yong et al. (2003b) and Meléndez \& Cohen (2007).

Relatively massive $\left(M>4 M_{\odot}\right)$ AGB stars can also contribute to the production of the heavy magnesium isotopes (e.g., Karakas \& Lattanzio 2003; Karakas 2010; Kobayashi et al. 2011). In such stars, there are a number of production sites: (1) the vicinity of the He-burning shell via $\alpha$-capture reactions on ${ }^{22} \mathrm{Ne}$, (2) via the $\mathrm{Mg}-\mathrm{Al}$ cycle in the vicinity of the H-burning shell, and (3) at the base of the convective envelope during the hot-bottom burning phase, again via the $\mathrm{Mg}-\mathrm{Al}$ cycle (Karakas
\& Lattanzio 2003). In all cases, the processed material can be brought to the surface layers and thus can contribute to the enrichment of the surrounding interstellar medium via mass loss in a stellar wind. As discussed in Karakas \& Lattanzio (2003) and Karakas (2010), the yields of ${ }^{25} \mathrm{Mg}$ and ${ }^{26} \mathrm{Mg}$ are complex functions of the initial abundance of the AGB star and of the parameters that control the thermal pulse structure, the extent of thermal dredge-up, the conditions in the convective envelope, and the rate of mass loss. In the context of the current work, it is worth noting that the production of ${ }^{25} \mathrm{Mg}$ and ${ }^{26} \mathrm{Mg}$ via $\alpha$-capture reactions on ${ }^{22} \mathrm{Ne}$ is unrelated to the abundance of aluminum. Consequently, since we find that the relative ${ }^{26} \mathrm{Mg}$ abundance is tightly coupled to that of $\mathrm{Al}$, at least for stars with $[\mathrm{Al} / \mathrm{Fe}] \gtrsim 0.5$ (see top panel of Figure 10 ), we can conclude that it is unlikely that the shell He-burning production site is a significant contributor. Indeed, it is likely that the primary nucleosynthesis site is hot-bottom burning in relatively massive AGB stars. 


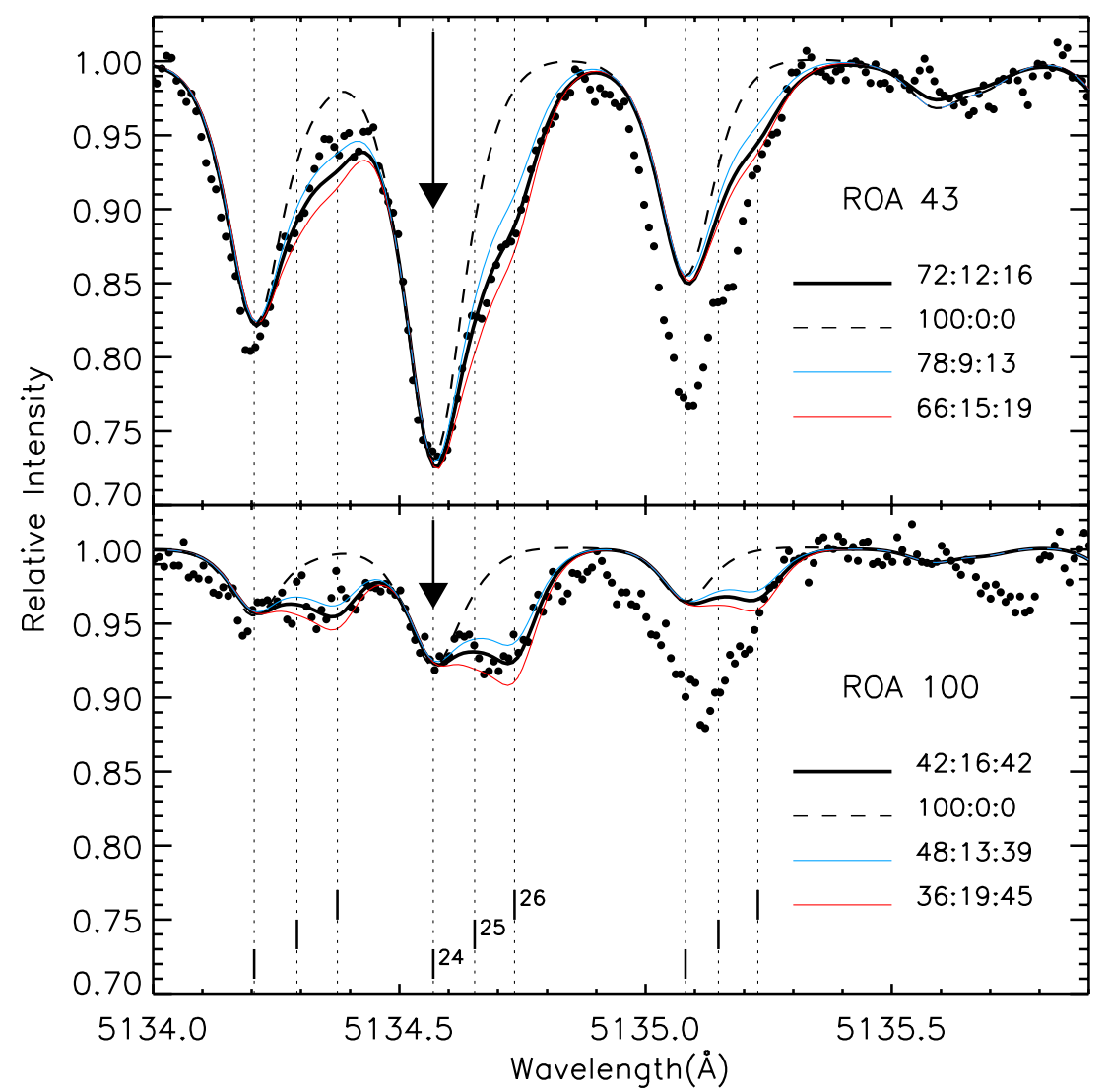

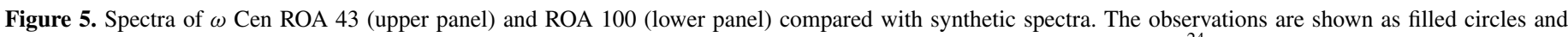

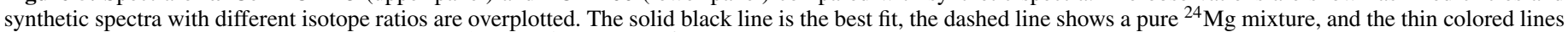
show unsatisfactory ratios. The locations of the ${ }^{24} \mathrm{MgH},{ }^{25} \mathrm{MgH}$, and ${ }^{26} \mathrm{MgH}$ lines are indicated. The $\mathrm{MgH}$ feature we are fitting is marked by the arrow.

(A color version of this figure is available in the online journal.)

In the panels of Figure 9, we plot the individual isotopic fractions relative to the total $\mathrm{Mg}$ abundance against $[\mathrm{Fe} / \mathrm{H}]$ for the stars observed in $\omega$ Cen, using the Norris \& Da Costa (1995b) $[\mathrm{Fe} / \mathrm{H}]$ values, and for the M4 stars using the cluster mean $[\mathrm{Fe} / \mathrm{H}]$ from Ivans et al. (1999). Also shown are the red giants observed in the globular clusters NGC 6752 (Yong et al. 2003a) and M13 and M71 (Yong et al. 2006). Since the observations in Yong et al. (2003a, 2006) were analyzed using very similar techniques to those employed here, no systematic effects are expected and the data can be combined. ${ }^{6}$ In the figure, filled symbols represent extreme population stars while open symbols represent primordial populations stars. Figure 10 shows the same relative abundance fractions but in this case plotted against the $[\mathrm{Al} / \mathrm{Fe}]$ abundance ratio.

Considering first the primordial stars in the middle and upper panels of Figure 9, there does not seem to be any obvious trend of increasing ${ }^{25} \mathrm{Mg}$ and ${ }^{26} \mathrm{Mg}$ fraction relative to total $\mathrm{Mg}$ with increasing $[\mathrm{Fe} / \mathrm{H}]$, although it may be that any (small) trend is disguised by the errors in the determinations. Indeed, the location of the primordial-star points in the lower panel of the figure, where effectively the ${ }^{25} \mathrm{Mg}$ and ${ }^{26} \mathrm{Mg}$ determinations are averaged, does support the existence of such a trend: making

\footnotetext{
6 We assume that the $[\mathrm{Fe} / \mathrm{H}]$ values are also free of systematic effects. This is supported to some extent by the fact the mean iron abundance found for NGC 6752 in Yong et al. (2003a), $[\mathrm{Fe} / \mathrm{H}]=-1.62$, is in reasonable accord with the mean iron abundance, $[\mathrm{Fe} / \mathrm{H}]=-1.52$, of the six NGC 6752 red giants analyzed in Norris \& Da Costa (1995b). Similarly, the $[\mathrm{Fe} / \mathrm{H}]$ determinations for M4 in Ivans et al. (1999) and Yong et al. (2008b) are in excellent agreement.
}

no attempt to distinguish ${ }^{25} \mathrm{Mg}$ from ${ }^{26} \mathrm{Mg}$, the relative isotopic ratios appear to change smoothly from $\sim 84: 7: 7$ at $[\mathrm{Fe} / \mathrm{H}] \approx$ -1.8 to $\sim 70: 15: 15$ at $[\mathrm{Fe} / \mathrm{H}] \approx-0.7$ dex. Such a trend is qualitatively consistent with the nucleosynthetic predictions of increasing ${ }^{25} \mathrm{Mg} /{ }^{24} \mathrm{Mg}$ and ${ }^{26} \mathrm{Mg} /{ }^{24} \mathrm{Mg}$ with $[\mathrm{Fe} / \mathrm{H}]$ (e.g., Kobayashi et al. 2011), although as noted in Yong et al. (2003a, 2006), for example, the isotopic ratios for these primordial globular cluster stars are significantly higher than for field stars of comparable $[\mathrm{Fe} / \mathrm{H}]$ abundance, despite being otherwise chemically similar. Yong et al. (2003a, 2006) postulate the existence of a generation of relatively massive AGB stars in the proto-cluster environment. These stars uniformly enrich the gas, from which the current primordial population stars form, in the heavy isotopes of $\mathrm{Mg}$ to a level above that generated by metalpoor supernovae, which is represented by the $\mathrm{Mg}$ isotopic ratios for the general halo field population at that metallicity. Given the similarity of our results for the $\omega$ Cen and M4 primordial stars to those of Yong et al. (2003a, 2006), it is apparent that this postulated scenario could also apply to the proto- $\omega$ Cen and the proto-M4.

Turning now to the full sample of stars, it is noteworthy from the middle panels of Figures 9 and 10 that there does not seem to be any obvious difference between the primordial and the extreme stars as regards the ${ }^{25} \mathrm{Mg}$ fractional abundance, regardless of $[\mathrm{Fe} / \mathrm{H}]$ and regardless of the $[\mathrm{Al} / \mathrm{Fe}]$ ratio. The lack of correlation between ${ }^{25} \mathrm{Mg}$ fractional abundance and [Al/Fe] has already been noted by Yong et al. (2003a, 2006) and it is apparent that the result applies to the $\omega$ Cen and M4 stars observed here as well. Further, the intermediate population 


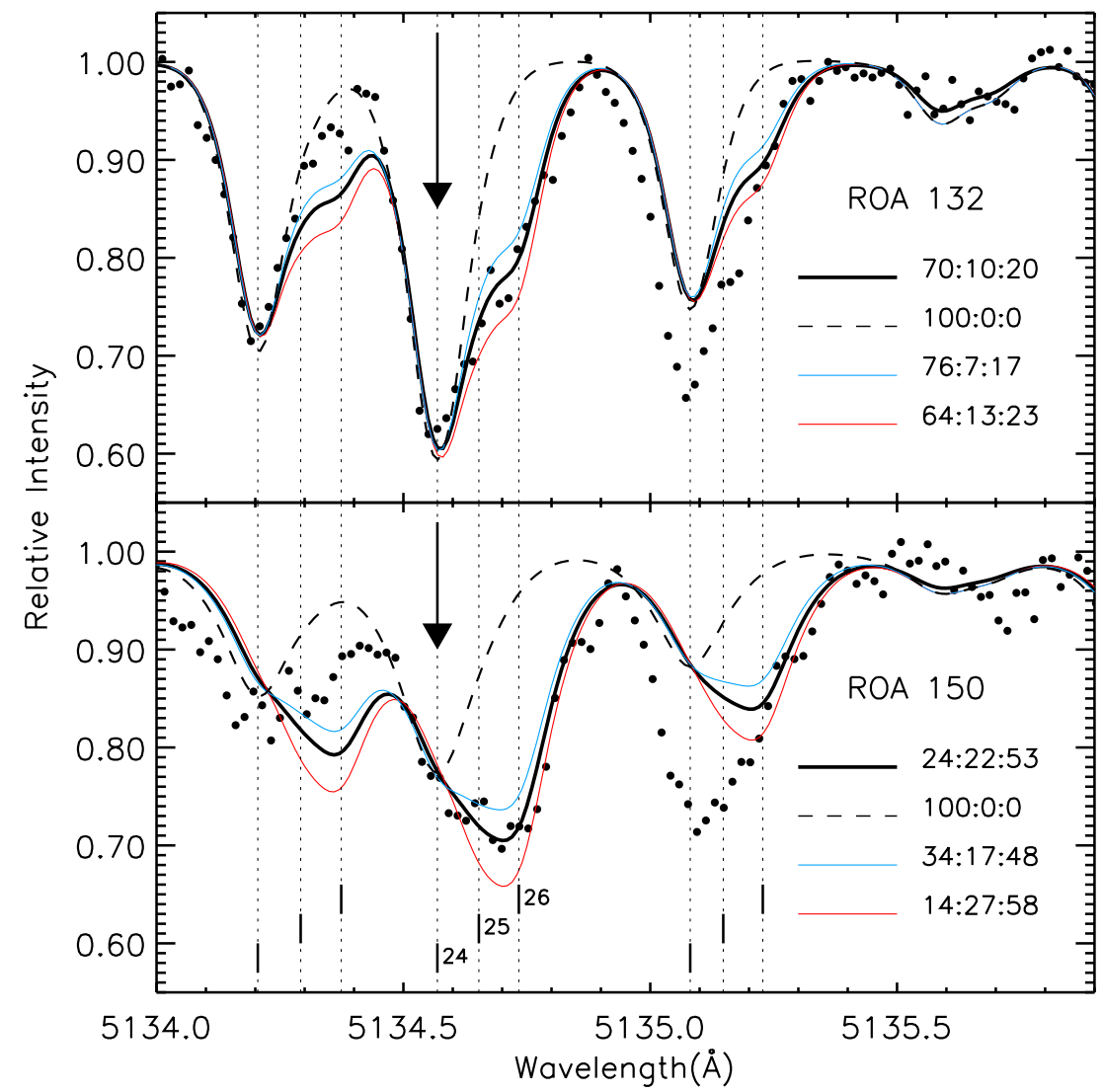

Figure 6. Same as for Figure 5 except for $\omega$ Cen stars ROA 132 (upper panel) and ROA 150 (lower panel). (A color version of this figure is available in the online journal.)

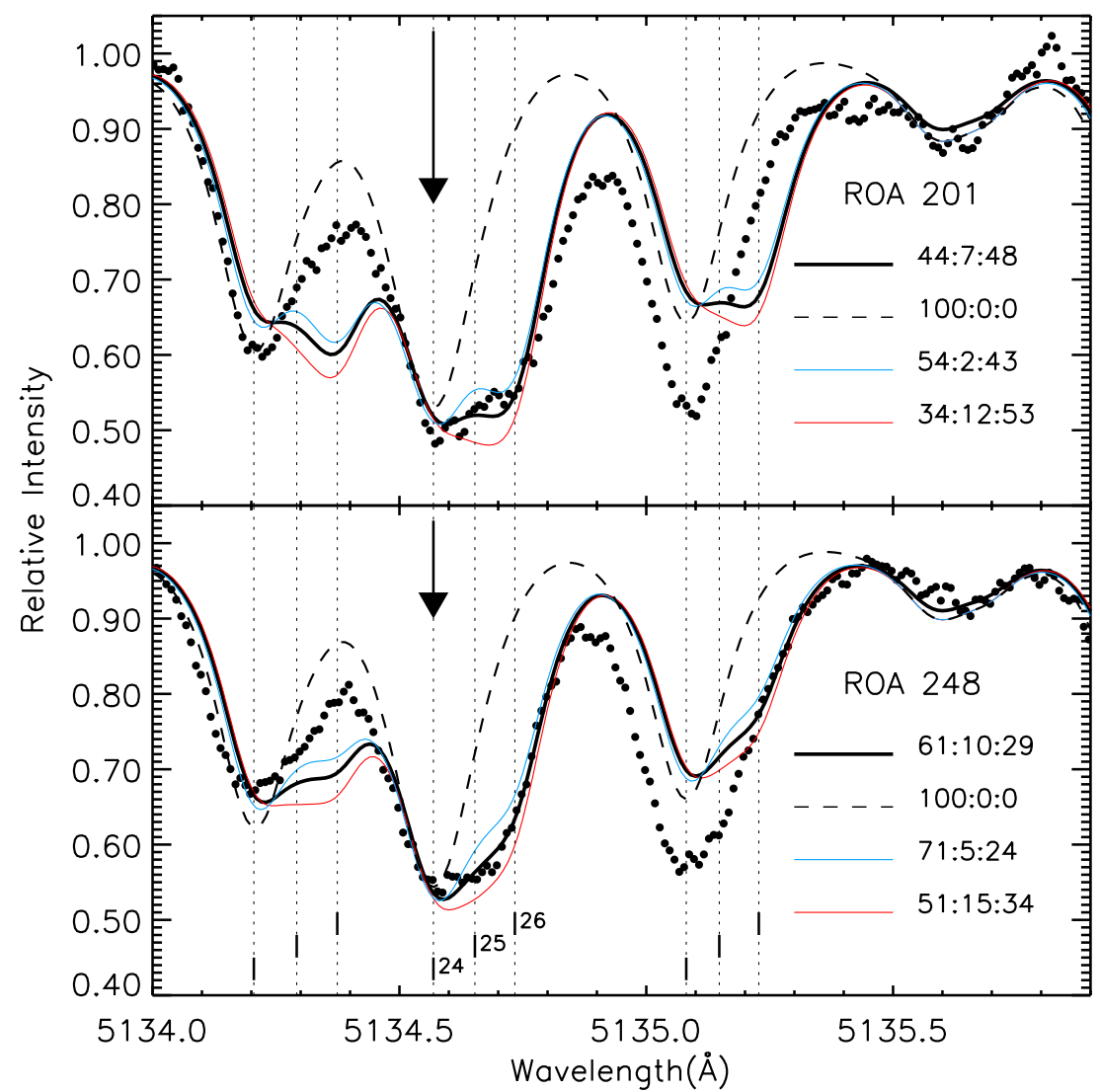

Figure 7. Same as for Figure 5 except for $\omega$ Cen stars ROA 201 (upper panel) and ROA 248 (lower panel). (A color version of this figure is available in the online journal.) 


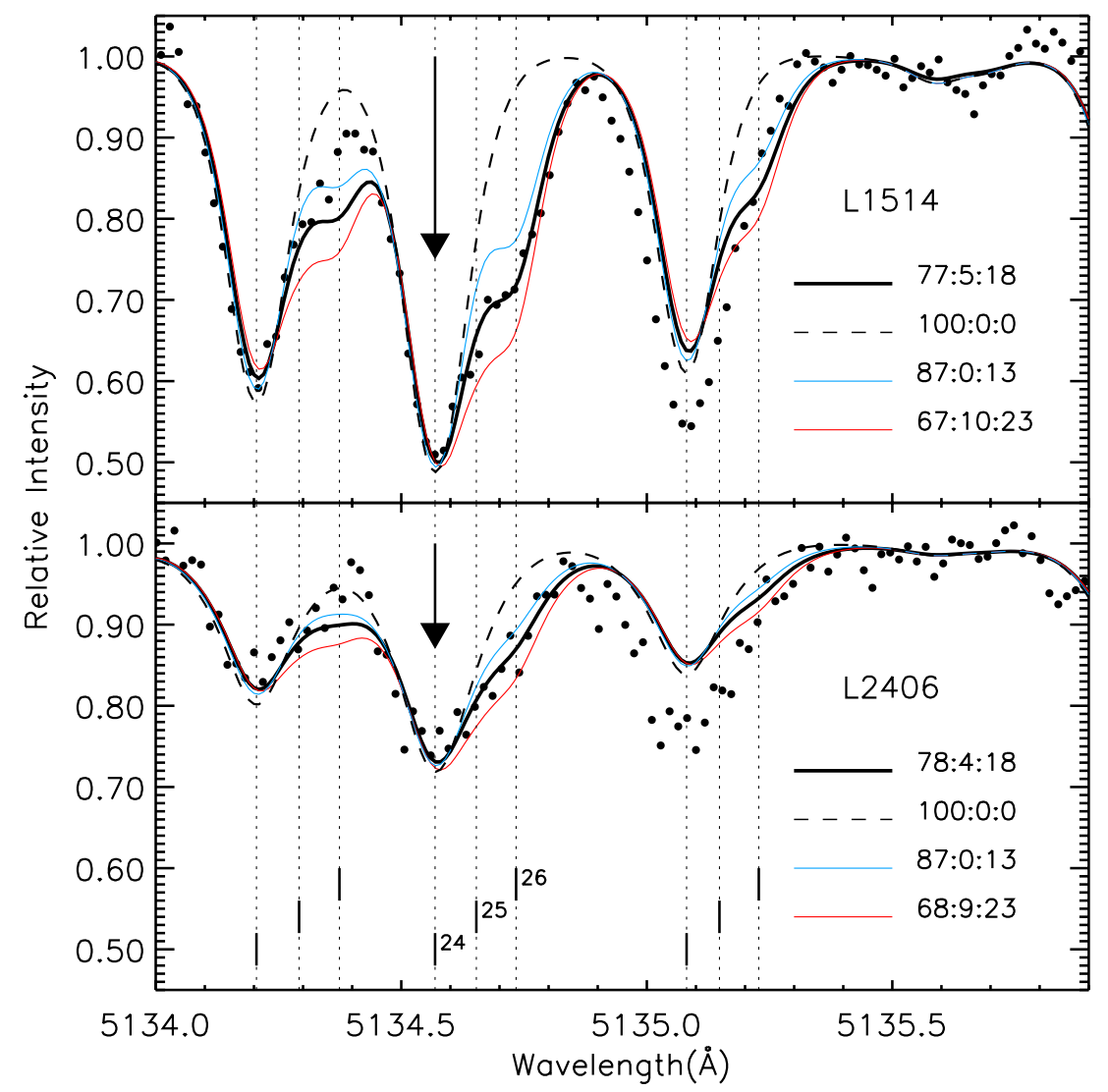

Figure 8. Same as for Figure 5 except for M4 stars L1514 (upper panel) and L2406 (lower panel). (A color version of this figure is available in the online journal.)

stars (predominantly from NGC 6752) also have similar values, and a single ${ }^{25} \mathrm{Mg}$ to total $\mathrm{Mg}$ fraction of order $10 \%$ appears consistent with all the determinations. Indeed for the 34 stars shown in the figures, the mean ${ }^{25} \mathrm{Mg}$ to total $\mathrm{Mg}$ fraction is $9.8 \%$ with a standard deviation of $4.6 \%$, which is comparable to the uncertainties in the individual determinations. In other words, in these globular cluster stars, which cover approximately $1 \mathrm{dex}$ in $[\mathrm{Fe} / \mathrm{H}]$ and $1.3 \mathrm{dex}$ in $[\mathrm{Al} / \mathrm{Fe}]$, and with the current uncertainties, the ${ }^{25} \mathrm{Mg}$ relative abundance appears not to be influenced by whether the star has been formed from the original cluster material, i.e., the primordial population, or from significantly "polluted" material as in the extreme population stars, which are presumed to incorporate material that has been processed through the $\mathrm{Mg}-\mathrm{Al}$ cycle. This lack of any obvious difference between the different populations is surprising as, a priori, one would not expect the relative ${ }^{25} \mathrm{Mg}$ abundance in $\mathrm{Mg}-\mathrm{Al}$ cycle processed material to be essentially identical to that for unprocessed material. In this context however, it is worth noting that Meléndez \& Cohen (2009) do find a small difference in the ${ }^{25} \mathrm{Mg}$ relative abundance between the $\mathrm{CN}$ weak (primordial) and the $\mathrm{CN}$-strong (intermediate) stars in the metal-rich globular cluster M71. Specifically, for five CN-weak stars, the mean value of the ${ }^{25} \mathrm{Mg}$ to total $\mathrm{Mg}$ relative abundance is $5.2 \% \pm 0.4 \%$, while for four CN-strong stars the mean ${ }^{25} \mathrm{Mg}$ relative abundance is slightly higher, $7.3 \% \pm 0.4 \%$. In both cases the error given is the standard error of the mean.

The situation for the ${ }^{26} \mathrm{Mg}$ fractional abundance, however, is markedly different. The upper panels (and, given the lack of variation in ${ }^{25} \mathrm{Mg}$, the effectively mirror images in the lower panels) of Figures 9 and 10 reveal clear dependences on both $[\mathrm{Fe} / \mathrm{H}]$ and on $[\mathrm{Al} / \mathrm{Fe}]$. In particular, Figure 9 shows that the production of ${ }^{26} \mathrm{Mg}$ is notably less at higher overall abundance. This is consistent with the fact that, in general, the extreme and intermediate population stars in globular clusters with larger $[\mathrm{Fe} / \mathrm{H}]$ values show lower enhancements in $[\mathrm{Al} / \mathrm{Fe}]$ compared to more metal-poor systems (e.g., Norris \& Da Costa 1995b; Yong et al. 2008b; Meléndez \& Cohen 2009; Johnson \& Pilachowski 2010; Da Costa \& Marino 2011; Carretta et al. 2013). This is usually interpreted (e.g., Johnson \& Pilachowski 2010) as a consequence of lower temperatures at the bottom of the convective envelope in the more metal-rich relatively massive AGB stars that are assumed to be the source of the $[\mathrm{Al} / \mathrm{Fe}]$ enhancements. Indeed, at least as regards $\omega \mathrm{Cen}$, D'Antona et al. (2011) claim that the smaller amount of $[\mathrm{Al} / \mathrm{Fe}]$ enhancement seen at higher $[\mathrm{Fe} / \mathrm{H}]$ values is consistent with the predictions of the Ventura \& D'Antona (2009) AGB star models. The reduced production of ${ }^{26} \mathrm{Mg}$ at larger $[\mathrm{Fe} / \mathrm{H}]$ seen in Figure 9 is then presumed to be consistent with the same interpretation, given the strong connection between the ${ }^{26} \mathrm{Mg}$ fractional abundance and $[\mathrm{Al} / \mathrm{Fe}]$ seen for the extreme stars in Figure 10.

As regards the data presented in the upper panel of Figure 10, and the implicit mirror image in the lower panel given the lack of variation in ${ }^{25} \mathrm{Mg}$, it appears that there are effectively two regions in the relation between ${ }^{26} \mathrm{Mg}$ and $[\mathrm{Al} / \mathrm{Fe}]$. The first is occupied by the primordial and intermediate population stars. Here, the $[\mathrm{Al} / \mathrm{Fe}]$ ratio increases by approximately 0.6 dex from the low value in the primordial stars but the ${ }^{26} \mathrm{Mg}$ fraction remains essentially constant, as does the ${ }^{24} \mathrm{Mg}$ fraction. The second region is occupied primarily by the extreme stars though it includes some intermediate populations stars. Here, we see that the ${ }^{26} \mathrm{Mg}$ abundance fraction is tightly coupled with that 

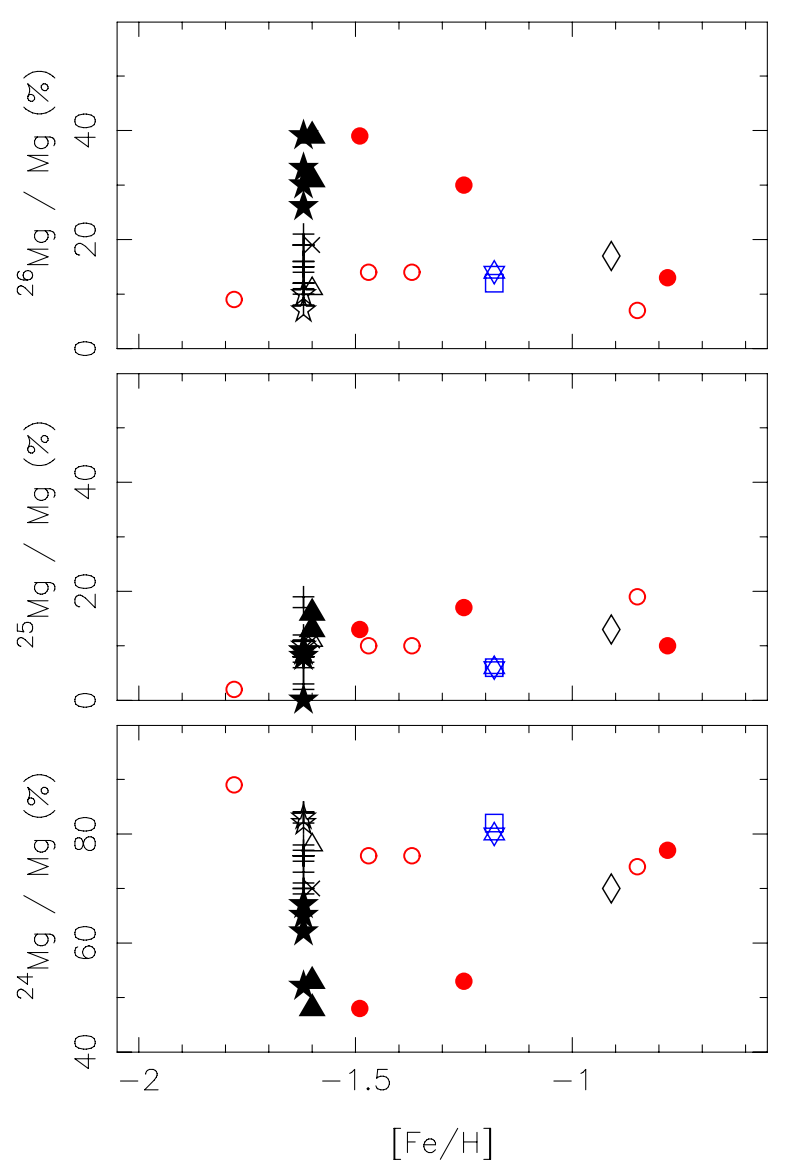

Figure 9. Relative abundance fractions for ${ }^{24} \mathrm{Mg}$ (lower panel), ${ }^{25} \mathrm{Mg}$ (middle panel), and ${ }^{26} \mathrm{Mg}$ (upper panel) compared to the total $\mathrm{Mg}$ abundance plotted against iron abundance $[\mathrm{Fe} / \mathrm{H}]$. Symbols are same as for Figure 4.

(A color version of this figure is available in the online journal.)

of ${ }^{27} \mathrm{Al}$ as the $[\mathrm{Al} / \mathrm{Fe}]$ ratio rises from $\sim 0.6$ dex to the value of $\sim 1.2-1.3$ in the most extreme population stars. At the same time, the ${ }^{26} \mathrm{Mg}$ fraction rises from approximately $10 \%$ to almost $40 \%$ while the ${ }^{24} \mathrm{Mg}$ fraction drops to $\sim 50 \%$.

The first region of the relation can be understood if it is assumed that the increased $\mathrm{Al}$ abundance is generated from ${ }^{24} \mathrm{Mg}$ via the $\mathrm{Mg}-\mathrm{Al}$ cycle. For example, if we assume the following parameters: $[\mathrm{Fe} / \mathrm{H}]=-1.62,[\mathrm{Mg} / \mathrm{Fe}]=+0.5,[\mathrm{Al} / \mathrm{Fe}]=0.0$, and $\mathrm{Mg}$ isotopic ratios of 80:10:10 (i.e., those of the NGC 6752 primordial stars), then, ignoring any dilution, converting just $10 \%$ of the ${ }^{24} \mathrm{Mg}$ atoms present to ${ }^{27} \mathrm{Al}$ results in an increase in $[\mathrm{Al} / \mathrm{Fe}]$ of 0.7 dex while the (total) $[\mathrm{Mg} / \mathrm{Fe}]$ is reduced by only 0.05 dex. Such a small change in $[\mathrm{Mg} / \mathrm{Fe}]$ is within the current limits on the variation in $[\mathrm{Mg} / \mathrm{Fe}]$ for the NGC 6752 primordial and intermediate population stars (Yong et al. 2003a). Further, assuming that the abundance of the more massive $\mathrm{Mg}$ isotopes remains relatively unaltered, then the isotopic ratios change only to $78: 11: 11$. Hence in this region of the diagram, the increase in $[\mathrm{Al} / \mathrm{Fe}]$ and the lack of any significant change in the isotope ratios are consistent with the conversion of a small amount of ${ }^{24} \mathrm{Mg}$ to $\mathrm{Al}$ in the $\mathrm{Mg}-\mathrm{Al}$ cycle. We note that the data shown in Figure 10 appear to require the consumption to be primarily of ${ }^{24} \mathrm{Mg}$; if the synthesized $\mathrm{Al}$ atoms in our example come entirely from ${ }^{25} \mathrm{Mg}$ or ${ }^{26} \mathrm{Mg}$ then that would produce significant changes in the ${ }^{25} \mathrm{Mg}$ or ${ }^{26} \mathrm{Mg}$ fractional abundances inconsistent with the observations shown in Figure 10.
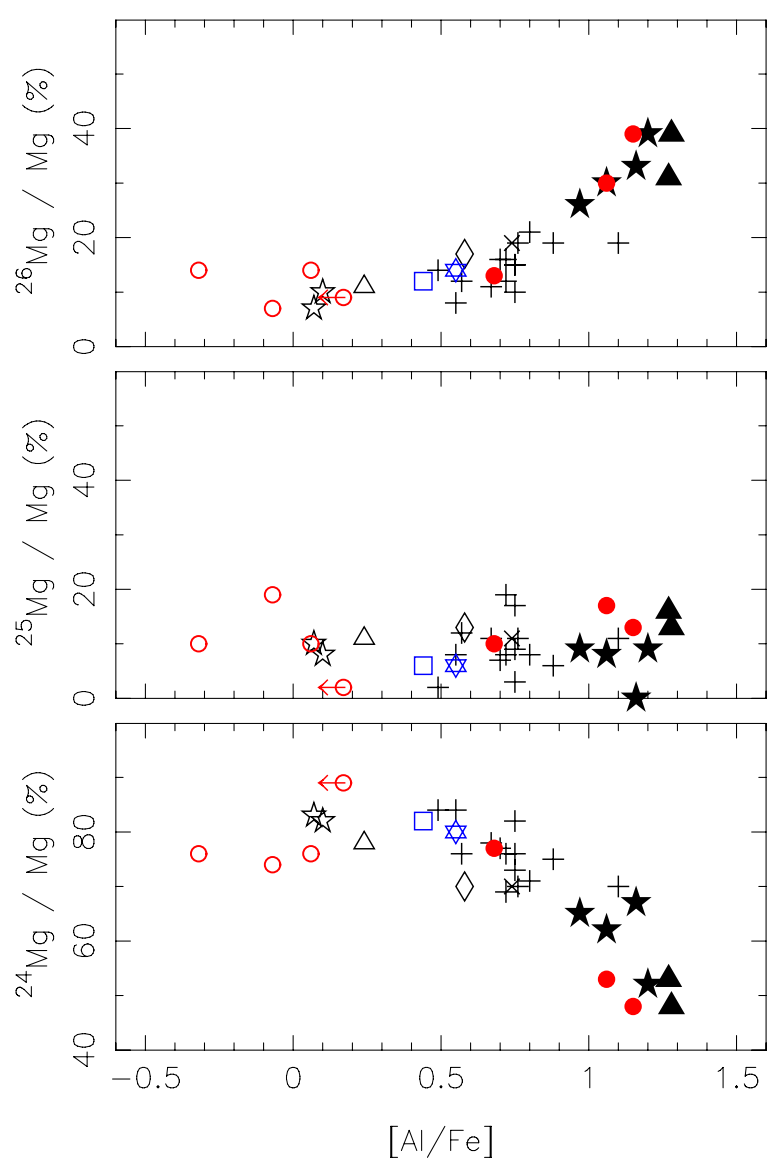

Figure 10. Relative abundance fractions for ${ }^{24} \mathrm{Mg}$ (lower panel), ${ }^{25} \mathrm{Mg}$ (middle panel), and ${ }^{26} \mathrm{Mg}$ (upper panel) compared to the total $\mathrm{Mg}$ abundance plotted against $[\mathrm{Al} / \mathrm{Fe}]$, the aluminum-to-iron abundance ratio. Symbols are same as for Figure 4.

(A color version of this figure is available in the online journal.)

In the second region of Figure 10, the production of $\mathrm{Al}$ and ${ }^{26} \mathrm{Mg}$ is apparently closely related. This is somewhat surprising, as in the conventional treatment of the $\mathrm{Mg}-\mathrm{Al}$ cycle, ${ }^{26} \mathrm{Mg}$ is destroyed by proton captures (to form ${ }^{27} \mathrm{Al}$ ) and is not expected to be made in any significant amount. Specifically, the protoncapture reaction on ${ }^{25} \mathrm{Mg}$ produces ${ }^{26} \mathrm{Al}$, which, in the ground state, has a long $\beta^{+}$-decay time of $\sim 0.72 \mathrm{Myr}$. Consequently, the cycle progresses from ${ }^{26} \mathrm{Al}$ primarily through the pathway ${ }^{26} \mathrm{Al}(p, \gamma){ }^{27} \mathrm{Si}\left(\beta^{+}\right){ }^{27} \mathrm{Al}$ and no ${ }^{26} \mathrm{Mg}$ is synthesized. ${ }^{26} \mathrm{Al}$ does, however, possess an excited state isomer, ${ }^{26} \mathrm{Al}^{m}$, for which the $\beta^{+}$-decay time is short $\left(6.3 \mathrm{~s}\right.$, comparable to the $\beta^{+}$-decay time of ${ }^{27} \mathrm{Si}$ ) thus providing a channel to produce ${ }^{26} \mathrm{Mg}$. Unfortunately, Iliadis et al. (2011) indicate that the rate for the reaction ${ }^{25} \operatorname{Mg}(p$, $\gamma)^{26} \mathrm{Al}^{m}$ is approximately a factor of five lower than the rate for the equivalent reaction involving the ground state of ${ }^{26} \mathrm{Al}$ at the temperatures likely to apply in the hot-bottom burning regions in relatively massive AGB stars. Consequently, the generation of ${ }^{27} \mathrm{Al}$ via the $\mathrm{Mg}-\mathrm{Al}$ cycle is not expected to show the strong correlation between ${ }^{27} \mathrm{Al}$ and ${ }^{26} \mathrm{Mg}$ seen in Figure 10.

In the context of the heuristic example outlined above, if we now suppose $50 \%$ (rather than $10 \%$ ) of the available $\mathrm{Mg}$ atoms are processed, including half of the original ${ }^{25} \mathrm{Mg}$ (but not ${ }^{26} \mathrm{Mg}$ ), and that the output of the processing is $80 \%{ }^{27} \mathrm{Al}$ and $20 \%$ ${ }^{26} \mathrm{Mg}$ (or equivalently $20 \%{ }^{26} \mathrm{Al}$ that is assumed to ultimately decay to ${ }^{26} \mathrm{Mg}$ ) then the resulting product has $[\mathrm{Mg} / \mathrm{Fe}]=0.28$, $[\mathrm{Al} / \mathrm{Fe}]=1.24$ and ${ }^{24} \mathrm{Mg},{ }^{25} \mathrm{Mg}$, and ${ }^{26} \mathrm{Mg}$ fractions of the total $\mathrm{Mg}$ abundance of $58 \%, 8 \%$, and $33 \%$, respectively. These 
values are consistent with the data of Figure 10 for the extreme stars. Moreover, the change in $[\mathrm{Mg} / \mathrm{Fe}]$ from +0.5 to +0.28 is consistent with the lower $[\mathrm{Mg} / \mathrm{Fe}]$ abundance ratios seen in the NGC 6752 extreme population stars (Yong et al. 2003a; see also Table 1). While these calculations are admittedly ad hoc, they do serve to illustrate that in order to understand the $\left({ }^{26} \mathrm{Mg},[\mathrm{Al} / \mathrm{Fe}]\right)$ relation revealed by the extreme population stars in Figure 10, synthesis of both ${ }^{27} \mathrm{Al}$ and ${ }^{26} \mathrm{Mg}$ from ${ }^{24} \mathrm{Mg}$ would seem to be required.

The discrepancy between the observational results presented in Figure 10 and current theory is highlighted by a comparison with the AGB star evolutionary models presented in Ventura et al. (2011). Their "reference model" is for a $6 M_{\odot}$ star with a composition typical of intermediate-metallicity globular clusters $([\alpha / \mathrm{Fe}]=+0.4$ and $Z=0.001$ or $[\mathrm{M} / \mathrm{H}] \approx-1.3)$ evolved through the complete AGB phase (see Ventura et al. 2011 for details). The temperature at the bottom of the convective envelope reaches sufficiently high values that the $\mathrm{Mg}-\mathrm{Al}$ cycle is initiated and over the course of the AGB evolution, i.e., prior to the loss of most of the envelope via mass loss, in the model the surface $\mathrm{Mg}$ abundance declines by about 0.35 dex while the surface $\mathrm{Al}$ abundance rises by $\sim 1$ dex, which is in approximate agreement with the observations. However, in this model ${ }^{26} \mathrm{Mg}$ is destroyed throughout the AGB evolution by the $p$-capture reaction and not synthesized, so that there is little real change, from its initial low value, in the ${ }^{26} \mathrm{Mg}$ fraction of the total surface $\mathrm{Mg}$ abundance (which decreases during the evolution). In contrast, with the reaction rates and the treatment of thermal pulses, third dredge-up and convective mixing used in the model (see Ventura et al. 2011 for details), the total surface $\mathrm{Mg}$ abundance becomes dominated by that of ${ }^{25} \mathrm{Mg}$, so that by the end of the evolution the ${ }^{25} \mathrm{Mg}$ fraction of the total $\mathrm{Mg}$ abundance exceeds $90 \%$ (Ventura et al. 2011). It is very difficult to see how these surface fractional abundances can be reconciled with the observations of the extreme population stars shown in Figure 10, even allowing for dilution of the AGB star surface abundances with pristine material, which is not depleted in overall $\mathrm{Mg}$ abundance and which has fractional isotopic abundances like those of the primordial stars.

We speculate here on two possibilities that might give rise to a higher ${ }^{26} \mathrm{Mg}$ fractional abundance in the most Al-rich stars assuming, given the strong correlation seen in Figure 10, that the general context is that of the $\mathrm{Mg}-\mathrm{Al}$ cycle and $\mathrm{AGB}$ star evolutionary calculations such as those of Ventura et al. (2011). In the first scenario, we postulate that the ${ }^{26} \mathrm{Mg}$ is synthesized as ${ }^{24} \mathrm{Mg}$ is depleted. To achieve this, we suggest, despite the results of Iliadis et al. (2011), an increase in the rate for the reaction ${ }^{25} \mathrm{Mg}(p, \gamma)^{26} \mathrm{Al}^{m}$ that produces the excited state isomer ${ }^{26} \mathrm{Al}^{m}$. As noted above, this isomer rapidly $\beta^{+}$-decays to produce ${ }^{26} \mathrm{Mg}$. An increase in this rate and in that for ${ }^{25} \mathrm{Mg}(p, \gamma)^{26} \mathrm{Al}^{g}$ would also decrease the ${ }^{25} \mathrm{Mg}$ fractional abundance reducing the "overproduction" of ${ }^{25} \mathrm{Mg}$ seen in the Ventura et al. (2011) models. In this scenario, the rate for the reaction ${ }^{26} \mathrm{Mg}(p, \gamma)^{27} \mathrm{Al}$ may also have to be reduced to ensure the synthesized ${ }^{26} \mathrm{Mg}$ is not entirely processed further to ${ }^{27} \mathrm{Al}$.

In the second scenario, we postulate that ${ }^{26} \mathrm{Al}$ (as distinct from ${ }^{26} \mathrm{Mg}$ ) is synthesized as ${ }^{24} \mathrm{Mg}$ is depleted. Here, we note that once $\mathrm{Mg}-\mathrm{Al}$ cycle processing has ceased, material rich in ${ }^{26} \mathrm{Al}$ will eventually show an increase in the ${ }^{26} \mathrm{Mg}$ relative abundance as the ${ }^{26} \mathrm{Al}$ radioactively decays to ${ }^{26} \mathrm{Mg}$. To achieve this, we suggest a significant increase in the ${ }^{25} \mathrm{Mg}(p, \gamma)^{26} \mathrm{Al}^{g}$ reaction rate (and probably also a reduction in the rate of the ${ }^{26} \mathrm{Al}(p, \gamma){ }^{27} \mathrm{Si}$ reaction, although ${ }^{27} \mathrm{Al}$ still needs to be produced from the $\beta^{+}$-decay of ${ }^{27} \mathrm{Si}$ ). Here, again in the context of the Ventura et al. (2011) models, the destruction of ${ }^{24} \mathrm{Mg}$ does not primarily produce ${ }^{25} \mathrm{Mg}$ but rather instead results in significant amounts of ${ }^{26} \mathrm{Al}$ (as well as ${ }^{27} \mathrm{Al}$ ). This "overproduced" ${ }^{26} \mathrm{Al}$ is then subsequently observed as ${ }^{26} \mathrm{Mg}$, the radioactive decay product of ${ }^{26} \mathrm{Al}$. The suggested rate increase would also serve to alleviate the discrepancy between the theoretical predictions and the observations as regards the ${ }^{25} \mathrm{Mg}$ relative abundance.

It is worth noting that the exploratory H-burning nucleosynthesis calculations of Prantzos et al. (2007), which were carried out at a variety of fixed temperatures rather than in the context of a full evolutionary stellar model, and which employed the best available reaction rate data, were able to reproduce the $\mathrm{Mg}$ isotope results for the globular cluster NGC 6752 given in Yong et al. (2003a) as well as the distributions of the O, $\mathrm{Na}$, and $\mathrm{Al}$ abundances. The agreement required quite specific conditions in their relatively simple analysis: a temperature of approximately $75 \mathrm{MK}$ (below $70 \mathrm{MK}{ }^{24} \mathrm{Mg}$ is hardly affected while above $80 \mathrm{MK}$ it is rapidly destroyed) and for the extreme stars, a dilution of the processed material via the incorporation of approximately $30 \%$ material having the primordial composition. They also point out that the "excess" of ${ }^{26} \mathrm{Mg}$ seen in the NGC 6752 stars can only be understood if it is originally produced as ${ }^{26} \mathrm{Al}$ (Prantzos et al. 2007). Whether these fixed temperature results apply to the more complex situation in real stars is a question that is beyond the scope of this paper.

We note only that our results for the $\mathrm{Mg}$ isotopic ratios in $\omega$ Cen extreme population stars reinforce the discrepancy between observations and stellar evolutionary theory already apparent in the results of Shetrone (1996) and Yong et al. (2003a, 2006). A resolution of the discrepancy is likely to lead to further insight into the complicated nucleosynthetic processes that occurred early in the lifetime of most globular clusters and in that respect further determinations of magnesium isotopic ratios in additional globular cluster stars would be very worthwhile. The data presented in Figures 9 and 10 suggests strongly that the stars to target in such a program, those most likely to have significantly non-solar $\mathrm{Mg}$ isotopic ratios, are the stars with the highest enhancements of $[\mathrm{Al} / \mathrm{Fe}]$ in globular clusters with intermediate or lower metallicities, i.e., $[\mathrm{Fe} / \mathrm{H}] \lesssim-1.4 \mathrm{dex}$.

G.S.D.C. and J.E.N. acknowledge the provision of travel funds for the Gemini-S observing run from the Australian Government Access to Major Research Facilities Program, grant 05/06-O-15. J.E.N. and D.Y. are also pleased to acknowledge support from Australian Research Council Discovery Projects grant DP0984924. The authors are grateful to Inese Ivans for carrying out some of the AAT observations and to the then Gemini-South Director Michael West for authorizing a classicalnight to queue-night swap when instrument problems looked likely to cause the complete loss of our second scheduled Gemini-S night. Thanks also to the Gemini staff for fixing the problems allowing completion of our program on the following night. The support of b-HROS scientist Steve Margheim during the run is also appreciated, as was the assistance of Sean Ryan during the original investigation of the suitability of UHRF for this observational program. Useful conversations with Amanda Karakas and John Lattanzio are also gratefully acknowledged.

This work is based in part on observations for program GS-2006A-Q-44 obtained at the Gemini Observatory, which is operated by the Association of Universities for Research in Astronomy, Inc., under a cooperative agreement with the 
NSF on behalf of the Gemini partnership: the National Science Foundation (United States), the Science and Technology Facilities Council (United Kingdom), the National Research Council (Canada), CONICYT (Chile), the Australian Research Council (Australia), Ministério da Ciência, Tecnologia e Inovação (Brazil), and Ministerio de Ciencia, Tecnología e Innovación Productiva (Argentina).

Facilities: AAT, Gemini:South

\section{REFERENCES}

Bekki, K., \& Freeman, K. C. 2003, MNRAS, 346, L11

Bevington, P. R., \& Robinson, D. K. 1992, Data Reduction and Error Analysis for the Physical Sciences (2nd ed.; New York, NY: McGraw-Hill)

Brown, J. A., \& Wallerstein, G. 1993, AJ, 106, 133

Caretta, E., Bragaglia, A., Gratton, R. G., et al. 2009, A\&A, 505, 117

Caretta, E., Bragaglia, A., Gratton, R. G., et al. 2010, A\&A, 516, A55

Caretta, E., Gratton, R., Bragaglia, A., D’Orazi, V., \& Lucatello, S. 2013, A\&A, 550, A34

Cavallo, R. M., Sweigart, A. V., \& Bell, R. A. 1996, ApJL, 464, L79

Da Costa, G. S., \& Marino, A. F. 2011, PASA, 28, 28

D’Antona, F., D'Ercole, A., Marino, A. F., et al. 2011, ApJ, 736, 5

de Mink, S. E., Pols, O. R., Langer, N., \& Izzard, R. G. 2009, A\&A, 507, L1

Decressin, T., Meynet, G., Charbonnel, C., Prantzos, N., \& Ekström, S. 2007, A\&A, 464, 1029

Denisenkov, P. A., \& Denisenkova, N. 1990, SvAL, 16, 275

Dinescu, D. I., Girard, T. M., \& van Altena, W. F. 1999, AJ, 117, 1792

Freeman, K. C. 1993, in ASP Conf. Ser. 48, The Globular Cluster-Galaxy Connection, ed. G. H. Smith \& J. P. Brodie (San Francisco, CA: ASP), 608 Gay, P. L., \& Lambert, D. L. 2000, ApJ, 533, 260

Iliadis, C., Champagne, A., Chieffi, A., \& Limongi, M. 2011, ApJS, 193, 16

Ivans, I. I., Sneden, C., Kraft, R. P., et al. 1999, AJ, 118, 1273

Johnson, C. I., \& Pilachowski, C. A. 2010, ApJ, 722, 1373

Johnson, C. I., Pilachowski, C. A., Simmerer, J., \& Schwenk, D. 2008, ApJ, 681,1505
Karakas, A. I. 2010, MNRAS, 403, 1413

Karakas, A. I., \& Lattanzio, J. C. 2003, PASA, 20, 279

Kobayashi, C., Karakas, A. I., \& Umeda, H. 2011, MNRAS, 414, 3231

Kurucz, R. L. 1996, in ASP Conf. Ser. 108, M.A.S.S.; Model Atmospheres and Spectrum Synthesis, ed. S. J. Adelman, F. Kupka, \& W. W. Weiss (San Francisco, CA: ASP), 2

Langer, G. E., Hoffman, R., \& Sneden, C. 1993, PASP, 105, 301

Marino, A. F., Milone, A. P., Piotto, G., et al. 2011, ApJ, 731, 64

Marino, A. F., Milone, A. P., Piotto, G., et al. 2012, ApJ, 746, 14

Marino, A. F., Villanova, S., Piotto, G., et al. 2008, A\&A, 490, 695

McWilliam, A., \& Lambert, D. L. 1988, MNRAS, 230, 573

Meléndez, J., \& Cohen, J. G. 2007, ApJL, 659, L25

Meléndez, J., \& Cohen, J. G. 2009, ApJ, 699, 2017

Norris, J. E. 2004, ApJL, 612, L25

Norris, J. E., \& Da Costa, G. S. 1995a, ApJL, 441, L81

Norris, J. E., \& Da Costa, G. S. 1995b, ApJ, 447, 680

Pancino, E., Mucciarelli, A., Sbordone, L., et al. 2011, A\&A, 527, A18

Persson, S. E., Frogel, J. A., Cohen, J. G., Aaronson, M., \& Matthews, K. 1980, ApJ, 235, 452

Piotto, G., Villanova, S., Bedin, L. R., et al. 2005, ApJ, 621, 777

Prantzos, N., Charbonnel, C., \& Iliadis, C. 2007, A\&A, 470, 179

Shetrone, M. D. 1996, AJ, 112, 2639

Smith, V. V., Suntzeff, N. B., Cunha, K., et al. 2000, AJ, 119, 1239

Sneden, C. 1973, ApJ, 184, 839

Timmes, F. X., Woosley, S. E., \& Weaver, T. A. 1995, ApJS, 101, 181

Ventura, P., Carini, R., \& D'Antona, F. 2011, MNRAS, 415, 3865

Ventura, P., \& D’Antona, F. 2005, ApJL, 635, L149

Ventura, P., \& D’Antona, F. 2009, A\&A, 499, 835

Woosley, S. E., \& Weaver, T. A. 1995, ApJS, 98, 617

Yong, D., Aoki, W., \& Lambert, D. L. 2006, ApJ, 638, 1018

Yong, D., Grundahl, F., Lambert, D. L., et al. 2003a, A\&A, 402, 985

Yong, D., Karakas, A. I., Lambert, D. L., Chieffi, A., \& Limongi, M. 2008a, ApJ, 689, 1031

Yong, D., Lambert, D. L., Allende Prieto, C., \& Paulson, D. B. 2004, ApJ, 603, 697

Yong, D., Lambert, D. L., \& Ivans, I. I. 2003b, ApJ, 599, 1357

Yong, D., Lambert, D. L., Paulson, D. B., \& Carney, B. W. 2008b, ApJ, 673,854 